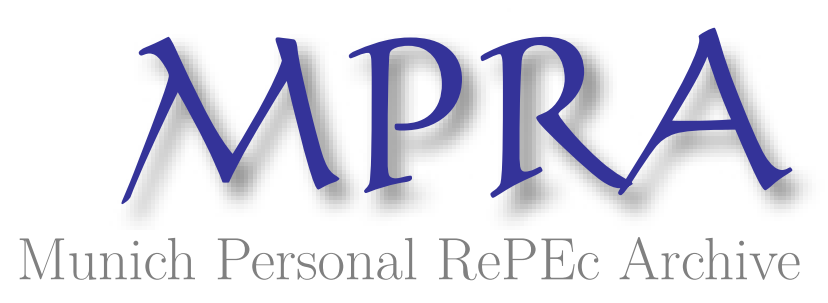

\title{
Performance Measurement in Multi-Task Agencies
}

\author{
Thiele, Veikko \\ University of British Columbia
}

31 March 2007

Online at https://mpra.ub.uni-muenchen.de/2469/

MPRA Paper No. 2469, posted 01 Apr 2007 UTC 


\title{
Performance Measurement in Multi-Task Agencies*
}

\author{
Veikko Thiele ${ }^{\dagger}$ \\ Sauder School of Business \\ University of British Columbia
}

March 31, 2007

\begin{abstract}
This paper analyzes a multi-task agency model with a risk-neutral and financially constrained agent. The agent's performance evaluation is thereby incongruent, i.e. it does not perfectly reflect the relative contribution of the agent's multi-dimensional effort to firm's profit. This paper elaborates on the improvement of the agent's performance evaluation through the costly acquisition of additional performance measures aimed at inducing the agent to implement a more efficient effort allocation across tasks. It contrasts two alternatives for the principal: $(i)$ to centrally invest in the information acquisition; or $(i i)$, to delegate this task to a supervisor. This paper demonstrates that the principal generally favors delegation for a sufficiently incongruent measurement of the agent's performance, and a centralized investment, otherwise.
\end{abstract}

Keywords: Multi-task agencies, performance measurement, distortion, congruity, limited liability, incentives.

JEL classification: D23, D82, M52

${ }^{*}$ I would like to thank Dominique Demougin, Gerald Feltham, Sandra Chamberlain, Carsten Helm, Christian Hofmann, Kay Mitusch, Anja Schöttner, Chee-Wee Tan, Louis-Philippe Sirois, Ralph Winter, participants of the 5th Symposium of the German Economic Association of Business Administration in Bonn and participants of the research workshop of the University of British Columbia for valuable comments and suggestions. Financial support by the Konrad-Adenauer Foundation is gratefully acknowledged and appreciated.

†Address: 2053 Main Mall, Vancouver, BC, Canada V6T 1Z2, phone: +1 604 822-3605, fax: +1 604 822-3163, e-mail: veikko.thiele@sauder.ubc.ca 


\section{Introduction}

Many employees are charged with performing multiple tasks which may differently contribute to firms' objectives. Employees can therefore not only decide on their effort intensity, but also on how to allocate their effort across all relevant tasks. If effort is non-contractible, firms face a two-dimensional incentive problem: they need not only to induce a sufficient effort intensity, but also to motivate an efficient effort allocation across tasks. The latter objective could be achieved if firms are able to identify each employee's individual contribution and apply this information in incentive contracts. However, company structures are often too complex for accrediting the respective contribution to each employee. As a consequence, firms are compelled to employ other potential incentive mechanisms.

One alternative is the application of objective performance measures in incentive contracts, as extensively analyzed in agency literature. ${ }^{1}$ However, the application of performance measures in incentive contracts can motivate employees to focus on activities which suitably enhance their performance evaluation, but have possibly little or even negative effects on firm value. The behavioral literature provides illustrative examples of such dysfunctional behavior. For instance, Prendergast [1999] reported that AT\&T rewarded their software engineers for the quantity of lines they wrote for their programs. It was soon discovered that the programs consisted of more lines than necessary.

Even if all relevant activities contribute to firm value, the relative effort allocation across those tasks can be inefficient. This occurs, when firms apply performance measures to provide their employees' with incentives, which do not perfectly reflect their individual contribution to firm value. The provision of incentives based on such incongruent performance measures leads therefore to a suboptimal effort allocation across tasks [Feltham and Xie, 1994]. For example, faculties at universities are primarily responsible for two tasks: teaching and conducting research. Since teaching is harder to quantify than the output from research, promotion decisions are generally made on the basis of research accomplishments. This in turn motivates particularly younger faculties to concentrate on research at the expense of teaching. ${ }^{2}$ Nevertheless, schools can modify their incentive schemes in order to improve the quality of teaching. Brickley and Zimmerman [2001] considered the incentive scheme adapted by the William E. Simon Graduate School of Business Administra-

\footnotetext{
${ }^{1}$ For a review of agency literature refer e.g. to Prendergast [1999], Lambert [2001], Gibbons [2005], and Christensen and Feltham [2005].

${ }^{2}$ See Kerr [1975] for a discussion of this and further examples. Additional illustrative examples are summarized by Gibbons [1998] and Baker [2000].
} 
tion, University of Rochester. After adjusting the performance evaluation and the reward system during the early 90 's, teaching quality improved significantly, but at the same time, research output declined.

If organizations do not have access to performance evaluations that are suitable to induce an efficient effort allocation, they need to apply alternative mechanisms that act to mitigate effort distortion. Recent multi-task agency literature analyzes and discusses some alternatives to improve agents' effort allocations. For instance, Feltham and Xie [1994], Banker and Thevaranjan [2000], and Datar, Kulp, and Lambert [2001] analyze, how multiple performance measures can be aggregated to mitigate effort distortion besides alleviating incentive risk. ${ }^{3}$ As demonstrated in Thiele [2006], the principal can even motivate the agent to implement non-distorted effort if she has access to a sufficient quantity of appropriate performance measures. A second stream of the multi-task agency literature focuses on the optimal design of jobs as a device to restrict effort distortion by allowing independent tasks to be split among multiple agents, see e.g. Holmström and Milgrom [1991], Schöttner [2005], Corts [2005], and Hughes, Zhang, and Xie [2005]. Finally, Holmström [1999] and Baker, Gibbons, and Murphy [2002] emphasize that transferring asset ownership to the agent can mitigate her effort distortion because asset ownership internalizes the consequences of her effort allocation. Nevertheless, such an approach compromises the objective of efficient risk-sharing if the agent is risk-averse and the principal is risk-neutral.

The ability to improve employees' performance evaluations in order to mitigate their effort distortion can be crucial for the efficiency of firms. To understand the provision of incentives in multi-task agency relations, it is essential to investigate whether and how organizations respond to the lack of perfectly congruent performance measures, and consequently, to the imposed inefficiencies due to effort distortion. Surprisingly, previous multi-task literature remains virtually silent about costly performance measurement with the objective of improving the agent's effort allocation. This paper thus focuses on costly performance measurement in order to glean new insights into the improvement of information systems aimed at mitigating effort distortion.

Specifically, I analyze a multi-task agency framework with risk-neutral parties, where the agent faces a liability limit constraint. Similar to Baker [2002], I adopt geometric representations for performance measure congruity, which eventually form the foundation for the considered measurement technology. The main emphasis lies in understanding the principal's preference for either

\footnotetext{
${ }^{3}$ For an empirical investigation on how performance measures are combined, refer to Gibbs, Merchant, Van der Stede, and Vargus [2004].
} 
investing centrally in assets which provides a viable means of measuring the agent's performance, or delegating the information acquisition to a supervisor. However, employing a supervisor induces a second moral hazard problem since the principal cannot contract over her effort to acquire information.

The analysis in this paper indicates that recruiting a supervisor for measuring the agent's performance can only be beneficial if she provides a sufficient relative measurement efficiency, which countervails the additionally imposed agency costs. More precisely, the principal's decision on whether to delegate the information acquisition to the supervisor is contingent on three factors: $(i)$ the precision of the supervisor's evaluation system, $(i i)$ the supervisor's comparative cost advantage in obtaining the required information; and $(i i i)$, the congruence of the costless available information system about the agent's effort. If the supervisor's performance evaluation is sufficiently precise, an adequately incongruent costless information system generally favors delegation. The rationale for this observation is that a less congruent costless information system imposes lower requirements on the supervisor's relative measurement efficiency, which is more likely to be satisfied by a potential supervisor. In contrast, a more congruent information system would impose higher requirements on the supervisor's comparative cost advantage, which is less likely to be achieved by a potential supervisor. In this case, a centralized investment is presumably to be observable. If the supervisor's performance evaluation is sufficiently imprecise, the contrary implications apply.

This paper is closely related to the multi-task agency literature analyzing the efficiency of induced effort allocations, especially to Holmström and Milgrom [1991], Feltham and Xie [1994], Datar et al. [2001], and Baker [2002]. However, it deviates in two main directions. First, it utilizes a framework with a risk-neutral and financially constrained agent, i.e. payments from the agent to the principal are not feasible. This allows to abstract from risk considerations in order to focus exclusively on the induced effort allocation. Second, the analyzed incentive contract is one of the bonus type similar to the ones applied by Park [1995], Kim [1997], Pitchford [1998] and Demougin and Fluet [2001] for single-task agency relations.

This paper contributes to the previous multi-task agency literature in two important ways. First, it extends their work by investigating a costly mechanism aimed at improving the agent's performance evaluation and hence, the efficiency of her motivated effort allocation. Second, this paper provides preliminary insights into the relationship between the properties of available information systems and the optimal design of organizations aimed at efficiently improving these information systems. 
The paper proceeds as follows. I introduce the basic model in section 2 and provide the firstbest solution in section 3. In section 4, I derive and discuss the second-best contract and elaborate on a ranking criteria for information systems in multi-task agencies with risk-neutral parties. Subsequently, I analyze in section 5 the principal's investment decision for generating additional measures about the agent's performance. Section 6 focuses on the contractual arrangement, when the principal employs a supervisor who is charged with the acquisition of the required performance measures. Both considered alternatives for improving the underlying information system are compared in section 7. Section 8 summarizes the main results and concludes.

\section{The Model}

Consider a single-period agency relationship between a principal and an agent. Both parties are risk-neutral and the agent faces a liability limit constraint, i.e. payment from the agent to the principal are not feasible. The agent is assigned to perform $n>2$ tasks which cannot be split among different agents. Therefore, she needs to implement a vector of effort $\boldsymbol{e}=\left(e_{1}, \ldots, e_{n}\right)^{t}$, $e \in \mathbf{E} \subseteq \mathbb{R}^{n+}$, where $e_{i}$ is the agent's effort allocated to task $i .^{4}$ Effort is non-verifiable and all activities $e_{i} \in \mathbf{E}$ are measured in the same unit. The agent's disutility of effort $C(\boldsymbol{e})$ is quadratic and separable in the different activities:

$$
C(\boldsymbol{e})=\sum_{i=1}^{n} \frac{1}{2} e_{i}^{2}=\frac{1}{2} \boldsymbol{e}^{t} \boldsymbol{e}
$$

By implementing effort $e$, the agent can affect the firm value $V$, which can be either high or low. Formally, let $V \in\{0,1\}$, whereas the probability for realizing the high firm value conditional on $\boldsymbol{e}$ is

$$
\operatorname{Prob}\{V=1 \mid \boldsymbol{e}\}=\min \left\{\boldsymbol{\mu}^{t} \boldsymbol{e}, 1\right\}
$$

Vector $\boldsymbol{\mu}=\left(\mu_{1}, \ldots, \mu_{n}\right)^{t}, \boldsymbol{\mu} \in \mathbb{R}^{n+}$, represents the sensitivity of the expected firm value in the agent's effort. Accordingly, the agent can not only influence the expected firm value by her effort intensity, but also by her relative effort allocation across relevant tasks. To ensure an interior solution for the first-best effort vector $\boldsymbol{e}^{f b}$, I assume that $\boldsymbol{\mu}$ is characterized such that $\operatorname{Prob}\left\{V=1 \mid \boldsymbol{e}^{f b}\right\}=\boldsymbol{\mu}^{t} \boldsymbol{e}^{f b}<1 .^{5}$

\footnotetext{
${ }^{4}$ All vectors are column vectors where ' $t$ ' denotes the transpose.

${ }^{5}$ By using the subsequently derived first-best solution, one can show that this requires $\boldsymbol{\mu}^{t} \boldsymbol{\mu}<1$.
} 
The realized firm value is non-contractible, and therefore, cannot be used to provide the agent with explicit incentives. Nevertheless, the principal receives a binary and verifiable signal $\bar{S} \in$ $\{0,1\}$, where $\bar{S}=1$ is the favorable signal in the sense of Milgrom [1981]. The probability of realizing the favorable signal is conditional on the agent's effort and takes the form

$$
\operatorname{Prob}\{\bar{S}=1 \mid \boldsymbol{e}\}=\min \left\{\overline{\boldsymbol{\omega}}^{t} \boldsymbol{e}, 1\right\}
$$

where $\overline{\boldsymbol{\omega}}=\left(\bar{\omega}_{1}, \ldots, \bar{\omega}_{n}\right)^{t}, \overline{\boldsymbol{\omega}} \in \mathbb{R}^{n+}$, represents the sensitivity of the expected signal in the agent's effort. This binary statistic potentially represents several costless performance measures which are summarized in the most efficient way. Henceforth, I refer to $\bar{S}$ as the costless information system. To ensure interior solutions, I shall assume that $\overline{\boldsymbol{\omega}}$ is characterized such that $\operatorname{Prob}\left\{\bar{S}=1 \mid \boldsymbol{e}^{*}\right\}=$ $\bar{\omega}^{t} e^{*}<1$ for the second-best effort vector $e^{*}{ }^{6}$

Since the realized value of $\bar{S}$ is verifiable, the principal can exploit this information in a bonus contract to provide the agent with incentives to implement effort. Particulary, the agent obtains a bonus $\beta^{A}$ in addition to a fixed transfer $\alpha^{A}$ if the favorable signal $\bar{S}=1$ is realized. Accordingly, the agent's binary wage $w^{A}$ takes the form

$$
w^{A}= \begin{cases}\alpha^{A}+\beta^{A}, & \text { if } \bar{S}=1, \\ \alpha^{A}, & \text { if } \bar{S}=0 .\end{cases}
$$

As a result of the agent's liability limit, all transfers have to be non-negative for any realization of $\bar{S}$. If the agent accepts this bonus contract on the basis of $\bar{S}$, it provides her with the expected utility

$$
U^{A}(\boldsymbol{e})=\alpha^{A}+\beta^{A} \overline{\boldsymbol{\omega}}^{t} \boldsymbol{e}-\frac{1}{2} \boldsymbol{e}^{t} \boldsymbol{e} .
$$

For parsimony, the agent's reservation utility is normalized to zero.

\section{The First-Best Contract}

Before investigating the second-best contract, it is useful to derive the first-best solution of this problem as a benchmark for the subsequent analysis. Suppose the principal can directly contract over $\boldsymbol{e}$. Then, she appoints the effort vector $e^{f b}$ which maximizes the difference between the expected firm value $\mathrm{E}[V \mid \boldsymbol{e}]$ and wage payment $w^{A}=C(\boldsymbol{e})$. Formally, the first-best effort vector $e^{f b}$ solves

$$
\max _{\boldsymbol{e}} \Pi(\boldsymbol{e}) \equiv \boldsymbol{\mu}^{t} \boldsymbol{e}-\frac{1}{2} \boldsymbol{e}^{t} \boldsymbol{e}
$$

\footnotetext{
${ }^{6}$ One can show by using the subsequently derived second-best solution that this requires $\boldsymbol{\mu}^{t} \overline{\boldsymbol{\omega}} / 2<1$.
} 
The first-order condition leads to $e^{f b}=\boldsymbol{\mu}$. The principal assigns each activity $e_{i}$ in accordance to its marginal effect $\mu_{i}$ on the expected firm value. To exemplify the relative effort allocation across tasks, consider the relation between two arbitrary activities $e_{i}^{f b}$ and $e_{j}^{f b}, i \neq j$ :

$$
\frac{e_{i}^{f b}}{e_{j}^{f b}}=\frac{\mu_{i}}{\mu_{j}} \quad i, j=1, \ldots, n, \quad i \neq j .
$$

Suppose $\mu_{i}>\mu_{j}$. In this case, it is optimal to assign more effort on task $i$ relative to task $j$ since the former task contributes more to the expected firm value than the latter. ${ }^{7}$ This implies that an implemented effort allocation is efficient if it reflects the relative marginal contribution of each task to the expected firm value. The conducted effort allocation is thus deemed to be non-distorted. In contrast, if an implemented effort allocation deviates from the one assigned under first-best, it is distorted. Formally, implemented effort is distorted if there exists no constant $\lambda>0$ satisfying $\boldsymbol{e}=\lambda \boldsymbol{e}^{f b}$.

Finally, substituting $\boldsymbol{e}^{f b}$ in the principal's objective function leads to

$$
\Pi^{f b}=\frac{1}{2} \boldsymbol{\mu}^{t} \boldsymbol{\mu} .
$$

The principal's expected first-best profit is therefore only characterized by the vector product $\boldsymbol{\mu}^{t} \boldsymbol{\mu}$, where $\boldsymbol{\mu}$ represents the sensitivity of the expected firm value in the agent's effort.

\section{The Second-Best Contract}

If the principal cannot directly contract over $e$, she faces an incentive problem. Since the firm value $V$ is non-contractible, she is compelled to use the information system $\bar{S}$ to provide the agent with incentives. However, the application of $\bar{S}$ in an incentive contract can motivate the agent to implement an inefficient effort allocation across tasks if $\mathrm{E}[\bar{S}]$ does not perfectly reflect her contribution to the expected firm value $\mathrm{E}[V]$. In this case, the information system $\bar{S}$ is deemed to be incongruent with the expected firm value, and its application in the agent's incentive contract imposes incongruent incentives.

In a second-best environment, the principal's problem is to find a bonus contract $\left(\alpha^{A *}, \beta^{A *}\right)$ aimed at maximizing her expected profit $\Pi \equiv \mathrm{E}\left[V-w^{A} \mid \boldsymbol{e}\right]$ while ensuring the agent's participation.

\footnotetext{
${ }^{7}$ Note that this observation applies because marginal effort costs are assumed to be identical across relevant tasks. If they differ, the task-specific marginal effort costs relative to the respective marginal contribution to firm value determine the first-best effort allocation, see Thiele [2006].
} 
Formally, the optimal bonus contract solves

$$
\max _{\alpha^{A}, \beta^{A}, \boldsymbol{e}} \Pi \equiv \boldsymbol{\mu}^{t} \boldsymbol{e}-\alpha^{A}-\beta^{A} \overline{\boldsymbol{\omega}}^{t} \boldsymbol{e}
$$

s.t.

$$
\begin{aligned}
& \alpha^{A}+\beta^{A} \overline{\boldsymbol{\omega}}^{t} \boldsymbol{e}-\frac{1}{2} \boldsymbol{e}^{t} \boldsymbol{e} \geq 0 \\
& \boldsymbol{e}=\arg \max _{\tilde{\boldsymbol{e}}} \alpha^{A}+\beta^{A} \overline{\boldsymbol{\omega}}^{t} \tilde{\boldsymbol{e}}-\frac{1}{2} \tilde{\boldsymbol{e}}^{t} \tilde{\boldsymbol{e}} \\
& \alpha^{A}+\beta^{A} \geq 0 \\
& \alpha^{A} \geq 0 .
\end{aligned}
$$

Condition (10) is the agent's participation constraint and ensures that it is in her interest to enter into this relationship. Moreover, (11) is the agent's incentive condition. Finally, (12) and (13) guarantee that the optimal bonus contract is compatible with the agent's liability limit.

We can directly infer from the agent's incentive constraint that she implements

$$
e^{*}=\beta^{A} \bar{\omega}
$$

The second-best effort vector consists of two components: the scalar $\beta^{A}$ and the vector $\boldsymbol{\mu}$. The bonus $\beta^{A}$ determines the overall effort intensity, whereas the relative effort allocation across tasks is characterized by $\overline{\boldsymbol{\omega}}$. To exemplify the effort allocation under second-best, consider again the relation between two arbitrary activities $e_{i}^{*}$ and $e_{j}^{*}, i \neq j$ :

$$
\frac{e_{i}^{*}}{e_{j}^{*}}=\frac{\bar{\omega}_{i}}{\bar{\omega}_{j}} \quad i, j=1, \ldots, n, \quad i \neq j .
$$

The agent places relatively more emphasis on tasks with a higher contribution to her performance evaluation in order to maximize the likelihood of obtaining the contracted bonus. From the principal's perspective, however, the motivated effort allocation is inefficient if it deviates from that implemented under first-best. Recall that the agent's effort allocation is distorted if there exists no constant $\lambda>0$ satisfying $\boldsymbol{e}^{*}=\lambda \boldsymbol{e}^{f b}$. Substituting $\boldsymbol{e}^{*}$ and $\boldsymbol{e}^{f b}$ leads to $\overline{\boldsymbol{\omega}} \beta=\lambda \boldsymbol{\mu}$. Apparently, effort distortion is rooted in the misalignment of the agent's performance evaluation with respect to firm value. To put it differently, effort distortion occurs if the agent's performance evaluation is incongruent, i.e. does not perfectly capture her contribution to firm value. In contrast, if the principal has access to a congruent information system, the agent can be motivated to implement the non-distorted (first-best) effort allocation. Formally, the costless information system is congruent if 
there exists a constant $\xi>0$ satisfying $\boldsymbol{\mu}=\xi \overline{\boldsymbol{\omega}}$. Otherwise, the agent's performance evaluation is incongruent and — when applied in an incentive contract—motivates her to implement an inefficient effort allocation across relevant tasks.

To exemplify the preceding observations, suppose the agent can implement two activities $e_{1}$ and $e_{2}$, where $e_{2}$ does not contribute to firm value $\left(\mu_{2}=0\right)$. Nonetheless, $e_{2}$ is suitable to positively influence her performance evaluation $\left(\bar{\omega}_{2}>0\right) .{ }^{8}$ Accordingly, it would be desirable from the principal's perspective to exclude $e_{2}$ from the performance measurement. However, since the performance evaluation is non-separable in the different activities, the principal is compelled to accept the implementation of $e_{2}$. This suggests two inefficiencies: First, the principal eventually rewards non-valuable activities $\left(e_{2}\right)$, and second, she needs to compensate the agent for the implementation of these activities in order to ensure her participation. However, even if $e_{2}$ contributes little to firm value relative to $e_{1}\left(\mu_{1}>\mu_{2}\right)$ but the expected signal is relatively more sensitive in $e_{2}$ than in $e_{1}$ $\left(\bar{\omega}_{1}<\bar{\omega}_{2}\right)$, the induced effort allocation is distorted as the agent inefficiently places more emphasis on tasks 2 than on task 1.

Proposition 1 The optimal bonus contract is characterized by $\alpha^{A *}=0$ and

$$
\beta^{A *}=\frac{\boldsymbol{\mu}^{t} \overline{\boldsymbol{\omega}}}{2 \overline{\boldsymbol{\omega}}^{t} \overline{\boldsymbol{\omega}}}
$$

The principal's expected second-best profit is

$$
\Pi^{*}=\frac{\left(\boldsymbol{\mu}^{t} \overline{\boldsymbol{\omega}}\right)^{2}}{4 \overline{\boldsymbol{\omega}}^{t} \overline{\boldsymbol{\omega}}}
$$

Proof First observe that the principal needs to set $\beta^{A}>0$ in order to ensure that the agent implements $e_{i}>0$ for at least one $i \in\{1, \ldots, n\}$. As a consequence, (12) is satisfied as long as (13) holds and therefore omits. Recall that $e^{*}=\beta^{A} \overline{\boldsymbol{\omega}}$. Thus, the Lagrangian becomes

$$
\mathcal{L}\left(\alpha^{A}, \beta^{A}\right)=\boldsymbol{\mu}^{t} \overline{\boldsymbol{\omega}} \beta^{A}-\alpha^{A}-\left(\beta^{A}\right)^{2} \overline{\boldsymbol{\omega}}^{t} \overline{\boldsymbol{\omega}}+\lambda\left[\alpha^{A}+\frac{1}{2}\left(\beta^{A}\right)^{2} \overline{\boldsymbol{\omega}}^{t} \overline{\boldsymbol{\omega}}\right]+\xi \alpha^{A} .
$$

The corresponding first-order conditions are

$$
\begin{gathered}
-1+\lambda+\xi=0, \\
\boldsymbol{\mu}^{t} \overline{\boldsymbol{\omega}}+\beta^{A} \overline{\boldsymbol{\omega}}^{t} \overline{\boldsymbol{\omega}}(\lambda-2)=0,
\end{gathered}
$$

\footnotetext{
${ }^{8}$ Feltham and Xie [1994] analyze a similar example in a setting, where the agent is risk-averse. They refer to this particular phenomenon as window dressing.
} 
and the complementary slackness conditions,

$$
\begin{gathered}
\lambda\left[\alpha^{A}+\frac{1}{2}\left(\beta^{A}\right)^{2} \overline{\boldsymbol{\omega}}^{t} \overline{\boldsymbol{\omega}}\right]=0 \\
\xi \alpha^{A}=0 .
\end{gathered}
$$

There are two cases to consider: $(i) \lambda>0$, and $(i i) \lambda=0$. First, suppose $\lambda>0$. In this case, $\alpha^{A}+\left(\beta^{A}\right)^{2} \overline{\boldsymbol{\omega}}^{t} \overline{\boldsymbol{\omega}} / 2=0$ due to (21). Since it is required that $\alpha^{A} \geq 0$, this would imply that $\alpha^{A *}=0$, $\beta^{A *}=0$, and consequently, $\boldsymbol{e}^{*}=(0, \ldots, 0)^{t}$. Therefore, $\lambda>0$ cannot be a solution of this problem. Thus, $\lambda=0$. Then, the second complementary slackness condition additionally implies that $\xi=1$. Consequently, $\alpha^{A *}=0$ due to (22). Solving (20) for $\beta^{A}$ with $\lambda=0$ leads to

$$
\beta^{A *}=\frac{\boldsymbol{\mu}^{t} \overline{\boldsymbol{\omega}}}{2 \overline{\boldsymbol{\omega}}^{t} \overline{\boldsymbol{\omega}}}
$$

Finally, the principal's expected profit can be obtained by substituting $\alpha^{A *}$ and $\beta^{A *}$ in the principal's objective function.

Q.E.D.

Observe that the optimal bonus $\beta^{A *}$ - and as a consequence the principal expected profit $\Pi^{*}$ depends on the relation between vector $\boldsymbol{\mu}$ and vector $\overline{\boldsymbol{\omega}}$. We can infer from preceding observations that the agent implements an inefficient effort allocation if $\boldsymbol{\mu}$ and $\overline{\boldsymbol{\omega}}$ are linearly independent. ${ }^{9}$ Baker [2002] demonstrated that performance measure congruity can be characterized by the angle between these two vectors. To provide a measure of the induced effort distortion and the corresponding efficiency loss, I subsequently adapt Baker's geometric interpretation to the underlying setting with a risk-neutral and financially constrained agent. This eventually provides the analytical foundation for the subsequently analyzed costly improvement of the agent's performance evaluation aimed at motivating a more efficient effort allocation.

Lemma 1 The angle $\bar{\varphi} \in[0, \pi / 2]$ between vector $\boldsymbol{\mu}$ and vector $\overline{\boldsymbol{\omega}}$ measures the induced effort distortion and associated efficiency loss if the bonus contract is dependent on $\bar{S} .{ }^{10}$

Proof See appendix.

This lemma indicates that the angle $\bar{\varphi}$ between the vector of the expected firm value sensitivities $\boldsymbol{\mu}$ and the vector of the expected signal sensitivities $\bar{\omega}$ measures not only the congruity of a

\footnotetext{
${ }^{9}$ Formally, vector $\boldsymbol{\mu}$ and vector $\overline{\boldsymbol{\omega}}$ are linearly independent if there exists no constant $\lambda>0$ satisfying $\boldsymbol{\mu}=\lambda \overline{\boldsymbol{\omega}}$.

${ }^{10}$ Throughout this paper, angles are represented in radian measures.
} 
performance measure as emphasized by Baker [2002], but also the induced effort distortion and the corresponding efficiency loss. ${ }^{11}$ The relation of these two vectors in the $n$-dimensional space is sufficient to characterize the inefficiency provoked by the application of an incongruent performance evaluation in the agent's incentive contract. Observe that the measure $\bar{\varphi}$ is negatively related to effort distortion and the corresponding efficiency loss. A smaller angle $\bar{\varphi}$ characterizes a more congruent information system, and therefore, induces less effort distortion. The provision of more congruent incentives is also desirable from the principal's perspective because it leads to a higher expected profit, as emphasized by lemma 1. To observe this, one can use the relations for vector products and re-write the principal's expected profit as

$$
\Pi(\bar{\varphi})=\frac{1}{4}\|\boldsymbol{\mu}\|^{2} \cos ^{2} \bar{\varphi}
$$

where $\|\boldsymbol{\mu}\|$ is the length of vector $\boldsymbol{\mu}$. Observe that the principal's expected profit is decreasing in $\bar{\varphi}$. The rationale for this observation is that a less congruent performance evaluation (higher $\bar{\varphi}$ ) motivates the agent to implement a less efficient effort allocation. This entails two inefficiencies: ( $i$ ) the expected firm value is less than it would have been under the implementation of less distorted effort; and ( $i i)$, the principal has to compensate the agent even for inefficiently chosen effort allocations in order to ensure her participation. Both effects deteriorate the efficiency of the bonus contract, and consequently, diminish the principal's expected profit.

Previous research dealing with single-task agency relations proposed several criteria to rank information systems with respect to their contract efficiency. ${ }^{12}$ However, these ranking criteria are in general not applicable when the agent's effort is multi-dimensional. If the agent is risk-averse, I have shown in Thiele [2006] that the relative value of information systems is determined by their respective congruency relative to their precision. In this framework, however, the agent is risk-neutral and uncertainty in her performance evaluation does not effect the contract efficiency. Accordingly, an information system is weakly superior to any other information system from the principal's perspective if it motivates a weakly more efficient effort allocation, and consequently, results in a weakly higher expected profit.

\footnotetext{
${ }^{11}$ The scaling of $\mathrm{E}[\bar{S} \mid \boldsymbol{e}]$ characterized by $\|\overline{\boldsymbol{\omega}}\|$ does not affect the efficiency of the bonus contract since the agent is risk-neutral. With risk-averse agents, however, the scaling is crucial since it affects the precision of performance measures and hence, the agent's required risk-premium, see e.g. Baker et al. [2002], Gibbons [2005], or Thiele [2006] for further discussions.

${ }^{12}$ For ranking of information systems in single-task agency relations with risk-averse agents, see e.g. Kim and Suh [1991]; and for a setting with a risk-neutral agent facing a liability limit constraint, see Demougin and Fluet [2001].
} 
Proposition 2 Suppose there exists a non-empty set of information systems $\mathbf{I}$ with $\bar{S}_{i} \in\{0,1\}$, $i \in \mathbf{I}$. Then, information system $k$ generating $\bar{S}_{k}, k \in \mathbf{I}$, is weakly superior to any other information system if and only if,

$$
\bar{\varphi}_{k} \leq \bar{\varphi}_{l}, \quad \forall l \in \mathbf{I}, l \neq k,
$$

where $\bar{\varphi}_{i}$ denotes the congruity measure for information system $i \in \mathbf{I}$.

The emphasized measure $\bar{\varphi}$ for the congruity of an information system and the induced effort distortion additionally allows us to rank information systems in multi-task agencies if all involved parties are risk-neutral. The superior information system—when applied in an incentive contractinduces the least effort distortion, and therefore, minimizes the efficiency loss for the principal.

Next, consider the congruity of $\bar{S}$ and its effect on the agent's expected utility. Substituting $\boldsymbol{e}^{*}$ with the optimal bonus contract $\left(\alpha^{A *}, \beta^{A *}\right)$ in (5) gives

$$
U^{A}(\bar{\varphi})=\frac{1}{8}\|\boldsymbol{\mu}\|^{2} \cos ^{2} \bar{\varphi}
$$

Obviously, the congruity measure $\bar{\varphi}$ additionally influences the agent's expected utility. The rationale for this observation is that the optimal bonus contract reflects the congruity of $\bar{S}$ in order to adjust the provision of incentives appropriately.

Proposition 3 The agent extracts a rent for all $\bar{\varphi} \in[0, \pi / 2)$. The rent is maximized for a congruent expected signal with $\bar{\varphi}=0$, and decreasing in $\bar{\varphi}$.

Proof The first derivative of $U^{A}(\bar{\varphi})$ with respect to $\bar{\varphi}$ gives

$$
\frac{\partial U^{A}(\bar{\varphi})}{\partial \bar{\varphi}}=-\frac{1}{4}\|\boldsymbol{\mu}\|^{2} \sin \bar{\varphi} \cos \bar{\varphi} .
$$

Since $2 \sin \bar{\varphi} \cos \bar{\varphi}=\sin (2 \bar{\varphi})$, this is equivalent to

$$
\frac{\partial U^{A}(\bar{\varphi})}{\partial \bar{\varphi}}=-\frac{1}{8}\|\boldsymbol{\mu}\|^{2} \sin (2 \bar{\varphi}),
$$

which is strictly negative for all $\bar{\varphi} \in(0, \pi / 2)$ due to the definition of the sine. Furthermore, $U^{A}(\bar{\varphi})$ is maximized for $\bar{\varphi}=0$, which implies $U^{A}(0)=\|\boldsymbol{\mu}\|^{2} / 8$. In contrast, $U^{A}(\pi / 2)=0$.

Q.E.D. 
Since the agent's liability limit is always binding (see proof of proposition 1), the principal must leave her a rent. More interestingly, however, is the observation that the agent extracts a higher rent, the more congruent the underlying information system is. This can be observed because providing the agent with more congruent incentives (lower $\bar{\varphi}$ ) leads to the implementation of less distorted effort. Then, it is beneficial from the principal's perspective to enhance the bonus aimed at motivating a higher effort intensity. To see this, one can re-write the optimal bonus as

$$
\beta^{A *}(\bar{\varphi})=\frac{\|\boldsymbol{\mu}\|}{2\|\overline{\boldsymbol{\omega}}\|} \cos \bar{\varphi}
$$

First observe that $\beta^{A *}(\bar{\varphi})$ is increasing in the performance measure congruity, i.e. $\bar{\varphi}$ decreases. As a result of the agent's liability limit, enhancing $\beta^{A *}(\bar{\varphi})$ contemporaneously leads to a higher rent extraction. However, if $\bar{\varphi}=\pi / 2$, the principal sets $\beta^{A *}(\pi / 2)=0$, thereby implying that the agent does not extract a rent. This observation is due to the fact that the application of $\bar{S}$ with $\bar{\varphi}=\pi / 2$ in a bonus contract would motivate the agent to implement an effort allocation which does not contribute to firm value. To see this, note that $\operatorname{Prob}\left\{V=1 \mid \boldsymbol{e}^{*}\right\}=\beta^{A}\|\boldsymbol{\mu}\|\|\overline{\boldsymbol{\omega}}\| \cos \bar{\varphi}$. Consequently, $\operatorname{Prob}\left\{V=1 \mid \boldsymbol{e}^{*}\right\}=0$ if $\bar{\varphi}=\pi / 2$.

\section{Costly Performance Measurement}

As the observations in preceding sections indicate, the contract efficiency in multi-task agencies is directly determined by the congruity of an available information system if all parties are riskneutral. Accordingly, the principal is better off if she has access to an information system that better reflects the agent's contribution to firm value, and can therefore be utilized to generate a more efficient effort allocation.

Feltham and Xie [1994] and Datar et al. [2001] have shown that the principal can mitigate effort distortion by combing multiple performance measures appropriately. Since they restricted their analysis to the aggregation of costless available performance measures, it is intuitive to expand on their initial theoretical foundation to focus on the next logical step of investigating investment decisions with the objective of improving the congruity of an information system. Suppose the principal centrally invests in assets which are suitable to generate additional measures about the agent's performance. The principal can therefore improve the congruity of the information system by incorporating the additionally acquired measures appropriately. The more measures generated, the more congruent will be the eventually established information system as a representation of all 
available performance measures. The principal's investment into the improvement of the information system is henceforth referred to as centralization.

To exemplify the underlying idea of costly performance measurement, consider a worker who is employed for producing goods. Suppose these goods achieve a higher price on the market, the better their quality is. The previous analysis indicates that the worker's incentive contract should incorporate certain quality measures to ensure the achievement of a desired quality level. In contrast to the produced quantity, however, verifying the quality is costly. If the firm wants to maintain a certain quality standard, it is necessary to invest in a quality verification mechanism, e.g. by acquiring a machine suitable to verify whether the quality of produced items is within a specified tolerance level. Both performance measures — produced quantity and achievement of a desired quality—can be appropriately combined and used to provide the worker with incentives. Particularly, the firm can commit to pay only a bonus if the produced quantity of items satisfying a desired quality exceeds a predefined number. This incentive scheme eventually ensures that the worker implements a more efficient effort allocation. Roughly speaking, the worker is now motivated to place more emphasis on the achievement of a certain quality standard besides producing a sufficient quantity.

Consider the principal's investment into the improvement of the agent's performance evaluation aimed at providing her with more congruent incentives. Let $m \in \mathbb{R}^{+}$be the measurement intensity, where a higher intensity generates a more congruent information system. The principal commits to a certain intensity $m$ by investing in the measurement system prior to negotiating with the agent about her incentive contract.

Assumption 1 The implementation of $m \in \mathbb{R}^{+}$generates a new verifiable and binary statistic $S(m) \in\{0,1\}$ characterized by

(i): $\operatorname{Prob}\{S=1 \mid m, \bar{\varphi}, \boldsymbol{e}\}=\min \left\{\boldsymbol{\omega}(\varphi(m, \bar{\varphi}))^{t} \boldsymbol{e}, 1\right\}$,

(ii): $\varphi(m, \bar{\varphi})<\bar{\varphi} \quad \forall m>0$.

The first condition emphasizes that the implemented measurement intensity $m$ generates a new information system which is represented by the binary statistic $S(m) \in\{0,1\}$. The corresponding expected signal is further characterized by the sensitivity vector $\boldsymbol{\omega}(\varphi(m, \bar{\varphi}))$. Its relation to the sensitivity vector $\boldsymbol{\mu}$ of the expected firm value is thereby determined by the angle $\varphi(\cdot)$, which measures the congruity of $S(m)$. The angle $\varphi(m, \bar{\varphi})$ as the congruity measure is a function of the implemented measurement intensity $m$ and the congruity measure $\bar{\varphi}$ of the costless available 


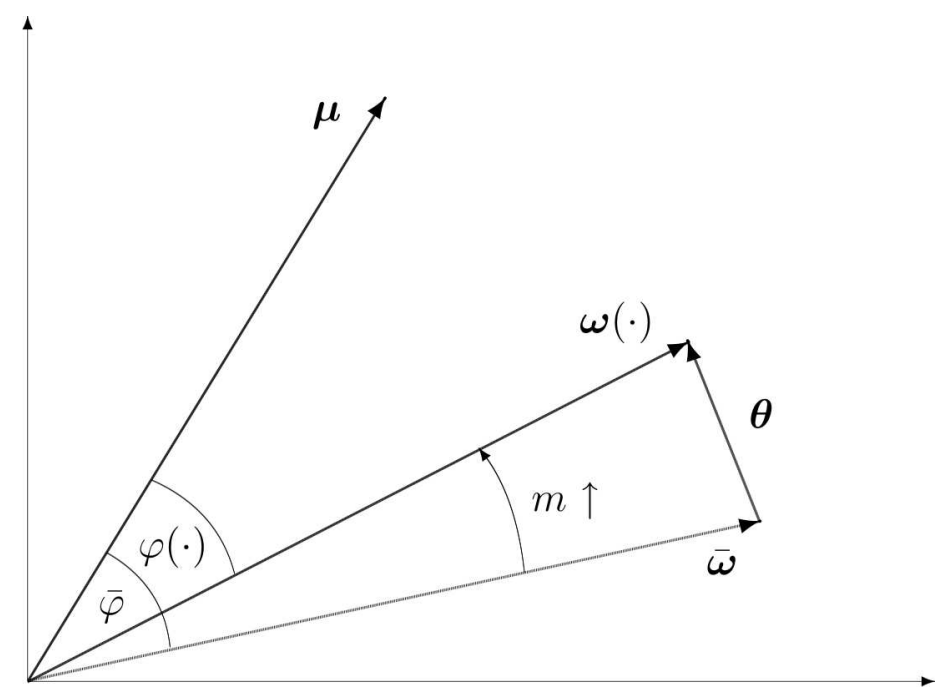

Figure 1: The Measurement Technology

information system $\bar{S}$. The final condition ensures that implementing a strictly positive intensity $m$ leads eventually to a more congruent information system. ${ }^{13}$

The underlying measurement technology is illustrated in figure 1 . The more intense the costly information acquisition $m$ is, the smaller becomes the angle between the vector of the expected firm value sensitivities $\boldsymbol{\mu}$ and the new vector of the expected signal sensitivities $\boldsymbol{\omega}(\cdot)$. Note that $\boldsymbol{\omega}(\cdot)$ is not necessarily on the plane spanned by $\boldsymbol{\mu}$ and $\overline{\boldsymbol{\omega}}$. It is only required that $\boldsymbol{\omega}(\cdot)$ is characterized by a smaller angle to $\boldsymbol{\mu}$ than vector $\overline{\boldsymbol{\omega}}$, i.e $\varphi(m, \bar{\varphi})<\bar{\varphi}$. Observe further that geometrically the new vector $\boldsymbol{\omega}(\cdot)$ is the sum of vector $\overline{\boldsymbol{\omega}}$ and vector $\boldsymbol{\theta}$, where $\boldsymbol{\theta}$ represents all information additionally acquired through costly performance measurement.

Recall that the optimal bonus $\beta^{A *}$ is normalized by $\|\bar{\omega}\|$ in order to exclude potential effects by different lengths of $\overline{\boldsymbol{\omega}}$. The same will be true if the length of $\boldsymbol{\omega}(\cdot)$ varies with $m$. For parsimony purposes and without loss of generality, I normalize the length of every new generated vector $\boldsymbol{\omega}(\cdot)$ to $\|\boldsymbol{\omega}(\cdot)\|=\|\overline{\boldsymbol{\omega}}\| .^{14}$

Assumption 2 The generated information system with $\varphi(m, \bar{\varphi})$ as its congruity measure is characterized by

(i): $\quad \varphi_{m}<0$ and $\varphi_{m m}>0 \forall m \geq 0$,

(ii): $\varphi_{\bar{\varphi}}>0$ and $\varphi_{m \bar{\varphi}}<0 \quad \forall m \geq 0$,

\footnotetext{
${ }^{13}$ Technically, the last condition requires that new generated performance measures are not sufficient statistics of other available measures.

${ }^{14}$ This assumption additionally ensures interior solutions, see section 2 .
} 
(iii): $\varphi(0, \bar{\varphi})=\bar{\varphi}$ and $\varphi(m, 0)=0$,

(iv): $\bar{\varphi} \in[0, \pi / 4]$,

where $\varphi_{i}$ denotes the first, and $\varphi_{i i}$ the second derivative of $\varphi(\cdot)$ with respect to $i, i=m, \bar{\varphi}{ }^{15}$

Condition (i) implies that a higher measurement intensity $m$ enhances the congruity of the generated information system ( smaller $\varphi(\cdot)$ ), whereas the marginal effect of reducing $\varphi(\cdot)$ is decreasing in $m$. Condition (ii) states that the new generated information system $S(m)$ is less congruent for a given measurement intensity $m$, the less congruent the costless information system $\bar{S}$ is. Moreover, the marginal effect of reducing $\varphi(\cdot)$ by implementing an arbitrary intensity $m$ is higher, the less congruent the costless information system is, i.e. the higher $\bar{\varphi}$ is. Condition (iii) emphasizes that without costly performance measurement, only the costless information system is available. Additionally, a perfectly congruent information system cannot be improved. To understand the last condition, observe from (24) that the expected gross benefit contingent on an arbitrary measurement intensity $m$ becomes

$$
\widehat{V}(m)=\frac{1}{4}\|\boldsymbol{\mu}\|^{2} \cos ^{2} \varphi(m, \bar{\varphi}) .
$$

It can be verified that $\widehat{V}(m)$ is strictly concave increasing in $m$ for $\varphi(\cdot) \in(0, \pi / 4)$, whereas the shape of $\widehat{V}(m)$ is ambiguous for $\varphi(\cdot) \in[\pi / 4, \pi / 2)$ and depends on the particular behavior of $\varphi(\cdot)$ in $m$. Consequently, condition (iv) guarantees that the first-order approach is sufficient for identifying the optimal measurement intensity.

Assumption 3 The implementation of $m \in \mathbb{R}^{+}$imposes costs $C(m)$ characterized by

(i): $\quad C^{\prime}(m)>0, C^{\prime \prime}(m)>0$, and $C^{\prime \prime \prime}(m) \geq 0$,

(ii): $\quad C(0)=C^{\prime}(0)=0$,

(iii): $\lim _{m \rightarrow \infty} C^{\prime}(m)=\infty$.

The first condition emphasizes that the investment costs for improving the agent's performance evaluation are strictly convex increasing in the conducted measurement intensity. The assumption about the third derivative is for technical reasons only. Condition $(i i)$ states that the principal bears no additional costs when she does not improve the information system. The final condition ensures

\footnotetext{
${ }^{15}$ For parsimony purposes, I suppress the arguments for the respective derivative, unless it is necessary for specific comparisons.
} 
an interior solution since the generation of a perfectly congruent information system is prohibitively costly. ${ }^{16}$

As explained earlier, the principal commits to a particular measurement intensity $m$ by investing $C(m)$ prior to negotiating with the agent about her bonus contracts. As a consequence, the agent's bonus contract is now on the basis of $S(m)$ and therefore, depends on $\varphi(m, \bar{\varphi})$, but everything else remains identical. Hence, we can directly turn to the principal's problem for identifying the optimal measurement intensity $m^{*}$. The adjustment of (24) with respect to the introduced measurement technology implies that the optimal intensity $m^{*}$ solves

$$
\max _{m} \Pi^{C}(m, \bar{\varphi})=\frac{1}{4}\|\boldsymbol{\mu}\|^{2} \cos ^{2} \varphi(m, \bar{\varphi})-C(m) .
$$

Since $2 \sin \varphi(\cdot) \cos \varphi(\cdot)=\sin (2 \varphi(\cdot))$, the first-order condition gives

$$
\frac{1}{4}\|\boldsymbol{\mu}\|^{2} \sin \left(2 \varphi\left(m^{*}, \bar{\varphi}\right)\right)\left(-\varphi_{m}\right)=C^{\prime}\left(m^{*}\right)
$$

The principal enhances the measurement intensity $m$ until the expected marginal gross benefit is equal to marginal costs. Since the expected gross benefit is concave, and investment costs are convex increasing in $m$, the first-order approach is also sufficient. ${ }^{17}$ Observe that the optimal measurement intensity $m^{*}$ depends implicitly on the congruity measure $\bar{\varphi}$ of the costless information system $\bar{S}$. The effect of $\bar{\varphi}$ on the optimal measurement intensity $m^{*}$ and the principal's expected profit $\Pi^{C}(\cdot)$ is clarified by the subsequent proposition.

Proposition 4 The optimal measurement intensity $m^{*}(\bar{\varphi})$ is increasing in $\bar{\varphi}$. Overall, the principal's expected profit $\Pi^{C}\left(m^{*}(\bar{\varphi}), \bar{\varphi}\right)$ decreases in $\bar{\varphi}$.

\footnotetext{
${ }^{16}$ This condition implicitly requires that the quantity of relevant tasks is strictly greater than the number of available performance measures that provide different information about the agent's effort allocation. For a formal analysis refer to Thiele [2006].

${ }^{17}$ To prove this analytically, observe that the second-order condition gives

$$
\frac{\partial^{2} \Pi^{C}(\cdot)}{\partial m^{2}}=\frac{1}{4}\|\boldsymbol{\mu}\|^{2}\left[2 \cos (2 \varphi(\cdot)) \varphi_{m}\left(-\varphi_{m}\right)+\sin (2 \varphi(\cdot))\left(-\varphi_{m m}\right)\right]-C^{\prime \prime}(m),
$$

which is equivalent to

$$
\frac{\partial^{2} \Pi^{C}(\cdot)}{\partial m^{2}}=-\frac{1}{4}\|\boldsymbol{\mu}\|^{2}\left[2 \cos (2 \varphi(\cdot))\left(\varphi_{m}\right)^{2}+\sin (2 \varphi(\cdot)) \varphi_{m m}\right]-C^{\prime \prime}(m) .
$$

Since $\cos (2 \varphi(\cdot))>0$ and $\sin (2 \varphi(\cdot))>0$ for all $\varphi(\cdot) \in(0, \pi / 4)$, the second derivative is strictly negative. Hence, the first-order approach is sufficient.
} 
Proof See appendix.

The less congruent the costless available information system $\bar{S}$ is (higher $\bar{\varphi}$ ), the more distorted would be the motivated effort allocation if the principal uses $\bar{S}$ to provide the agent with incentives. Since this diminishes the principal's expected profit, it is optimal from her perspective to invest more in the improvement of the information system aimed at mitigating effort distortion. However, improving the information system imposes strictly convex increasing costs. Consequently, the additional inefficiency due to an increases of $\bar{\varphi}$ cannot be compensated by enhancing $m^{*}(\cdot)$. As a result, $\Pi^{C}(\cdot)$ decreases in $\bar{\varphi}$.

By committing to the optimal measurement intensity $m^{*}(\cdot)$, the principal ensures that the agent's performance evaluation is eventually characterized by a desired congruity measure $\varphi\left(m^{*}(\cdot), \bar{\varphi}\right)$. This in turn motivates the agent to choose a more efficient effort allocation in comparison to the application of $\bar{S}$. The preceding analysis indicates that the agent's bonus contract reflects the congruity of her performance evaluation. To illustrate the effect of improving the incentive congruity on the agent's expected utility, observe that $U^{A}(\cdot)$ changes to

$$
U^{A}\left(m^{*}(\bar{\varphi}), \bar{\varphi}\right)=\frac{1}{8}\|\boldsymbol{\mu}\|^{2} \cos ^{2} \varphi\left(m^{*}(\bar{\varphi}), \bar{\varphi}\right) .
$$

Recall that $2 \sin (\cdot) \cos (\cdot)=\sin (2(\cdot))$. Consequently, the first derivative of $U^{A}(\cdot)$ with respect to $m$ is

$$
\frac{\partial U^{A}(\cdot)}{\partial m}=\frac{1}{8}\|\boldsymbol{\mu}\|^{2} \sin (2 \varphi(\cdot))\left(-\varphi_{m}\right),
$$

which is strictly positive for all $\varphi(\cdot) \in(0, \pi / 2)$ since $\varphi_{m}(\cdot)<0$. Accordingly, improving the information system contemporaneously implies a higher rent extraction by the agent. This in turn constitutes indirect costs for mitigating effort distortion if the agent faces a liability limit constraint.

\section{Delegation}

Instead of centrally investing in the improvement of the agent's performance evaluation, the principal can alternatively delegate the information acquisition to a supervisor. This can be favored by the principal if the supervisor is able to measure the agent's performance more efficiently than a centralized regime. However, employing a supervisor with the objective of improving the information system imposes a second moral hazard problem. Thus, the principal needs to provide the supervisor with appropriate incentives in order to ensure the implementation of a desired measurement intensity. 
Suppose the principal recruits a risk-neutral supervisor who also faces a liability limit constraint. For parsimony, her reservation utility is normalized to zero. The supervisor can conduct a performance measurement with the intensity $m^{S} \in \mathbb{R}^{+}$satisfying assumptions 1 and 2. A strictly positive measurement intensity therefore generates additional measures about the agent's performance, which can be used to enhance the congruence of her performance evaluation. The supervisor's performance measurement is therefore objective rather than subjective. ${ }^{18}$ Recall the quantity/quality example from section 5. Instead of purchasing a machine verifying the achievement of a quality standard, the supervisor may verify and document the quality characteristics of produced items. Both information - the produced quantity and achieved quality - can be appropriately combined to provide the agent with more congruent incentives. Finally, the manipulation of generated performance measures is assumed to be prohibitively costly for the supervisor.

For the sake of comparability, the supervisor's disutility of conducting an arbitrary measurement intensity $m^{S}$ is $C^{S}\left(m^{S}\right)=\eta C\left(m^{S}\right)$, where $C\left(m^{S}\right)$ is identical to the principal's investment costs for all $m^{S}=m$. Potential differences in the measurement efficiency are characterized by $\eta \in \mathbb{R}^{+}$. The ratio $1 / \eta$ thereby measures the supervisor's comparative advantage in generating the same performance measures relative to the principal's central investment.

The principal observes the realization of the binary statistic $S\left(m^{S}\right) \in\{0,1\}$ but cannot directly contract over $m^{S}$. Suppose the unfavorable signal $S\left(m^{S}\right)=0$ is realized. This can occur because either the agent indeed failed to meet her performance objective, or the supervisor did not generate the required information such that there exists no evidence about the implementation of the desired effort allocation. Consequently, the principal needs to provide the supervisor with incentives to motivate a desired measurement intensity $m^{S}>0$. The principal, however, receives several biased information about the conducted measurement intensity, which are summarized by the binary statistic $M \in\{0,1\}{ }^{19}$

Assumption 4 Let $\operatorname{Pr}\left[M=1 \mid m^{S}\right]=\rho\left(m^{S}\right) \in[0,1)$, where the probability $\rho\left(m^{S}\right)$ to realize the favorable signal $M=1$ satisfies

$$
\text { (i): } \quad \rho(0)=0 \text {, }
$$

\footnotetext{
${ }^{18}$ Subjective evaluations imply additional problems since implicit promises to reward favorable behavior needs to be self-enforcing in repeated games [Baker, Gibbons, and Murphy, 1994], or induce favoritism by the evaluator [Prendergast and Topel, 1996].

${ }^{19}$ See Demougin and Fluet [2001] for a formal derivation that verifiable information about one-dimensional effort can be summarized by a binary statistic if parties are risk-neutral.
} 
(ii): $\quad \rho\left(m^{S}\right)$ is twice-continuously differentiable with $\rho^{\prime}\left(m^{S}\right)>0$ and

$$
\rho^{\prime \prime}\left(m^{S}\right) \leq 0 \forall m^{S} \geq 0,
$$

(iii): $\lim _{m^{S} \rightarrow \infty} \rho\left(m^{S}\right)=1$ and $\lim _{m^{S} \rightarrow 0} \rho^{\prime}\left(m^{S}\right)=\infty$.

Condition (i) emphasizes that without improving the information system, the binary statistic $M$ can never be favorable. The second and third conditions are standard and guarantee an interior and unique solution for the optimal measurement intensity.

In order to induce a desired measurement intensity, the principal provides the supervisor with a bonus contract $w^{S}$ on the basis of the verifiable statistic $M$. In particular, let

$$
w^{S}= \begin{cases}\alpha^{S}+\beta^{S}, & \text { if } \quad M=1, \\ \alpha^{S}, & \text { if } \quad M=0,\end{cases}
$$

where $\alpha^{S}$ denotes the fixed transfer and $\beta^{S}$ the bonus payment. The supervisor can increase the probability $\rho\left(\mathrm{m}^{S}\right)$ to realize the favorable signal $M=1$, and consequently, the likelihood to obtain the contracted bonus $\beta^{S}$, when she enhances her measurement intensity $m^{S}$. Since the supervisor is risk-neutral, the bonus contract provides her with the expected utility

$$
U^{S}\left(m^{S}\right)=\alpha^{S}+\rho\left(m^{S}\right) \beta^{S}-\eta C\left(m^{S}\right) .
$$

Before deriving the optimal bonus contract for the supervisor, it is essential to briefly consider the timing of this problem. First, the principal offers the supervisor a bonus contract $w^{S}$. If she accepts the contract and participates, it provides her with incentives to implement a desired measurement intensity $m^{S *}$. Afterwards, the principal provides the agent with a bonus contract on the basis of her anticipated performance evaluation represented by the binary statistic $S\left(m^{S *}\right)$. Then, the agent implements effort and the supervisor subsequently generates the additional performance measure(s). Afterwards, the agent's and supervisor's performance evaluations $S\left(m^{S *}\right)$ and $M$ are realized and become public knowledge. Finally, all contracted payments take place.

The agent's bonus contract depends now on the supervisor's induced measurement intensity $m^{S *}$, but remains qualitatively the same as in section 5 . Therefore, we can directly turn to the derivation of the supervisor's contract, which is aimed at maximizing the principal's expected profit while guaranteeing the supervisor's participation. Formally, the supervisor's optimal bonus contract solves 


$$
\max _{\alpha^{S}, \beta^{S}, m^{S}} \Pi^{D} \equiv \frac{1}{4}\|\boldsymbol{\mu}\|^{2} \cos ^{2} \varphi\left(m^{S}, \bar{\varphi}\right)-\alpha^{S}-\rho\left(m^{S}\right) \beta^{S}
$$

s.t.

$$
\begin{aligned}
& \alpha^{S}+\rho\left(m^{S}\right) \beta^{S}-\eta C\left(m^{S}\right) \geq 0 \\
& m^{S}=\arg \max _{\tilde{m}^{S}} \alpha^{S}+\rho\left(\tilde{m}^{S}\right) \beta^{S}-\eta C\left(\tilde{m}^{S}\right) \\
& \alpha^{S}+\beta^{S} \geq 0 \\
& \alpha^{S} \geq 0 .
\end{aligned}
$$

Condition (40) is the supervisor's participation constraint and guarantees that it is in her interest to enter into this relationship. Additionally, (41) is the supervisor's incentive constraint. Finally, conditions (42) and (43) ensure that the bonus contract is compatible with the supervisor's liability limit.

Proposition 5 The supervisor's optimal bonus contract is characterized by the fixed transfer $\alpha^{S *}=$ 0 and the expected bonus

$$
B\left(m^{S *}, \eta\right)=\frac{\eta C^{\prime}\left(m^{S *}\right) \rho\left(m^{S *}\right)}{\rho^{\prime}\left(m^{S *}\right)} .
$$

The optimal measurement intensity $m^{S *}$ thereby solves

$$
\frac{1}{4}\|\boldsymbol{\mu}\|^{2} \sin \left(2 \varphi\left(m^{S *}, \bar{\varphi}\right)\right)\left(-\varphi_{m}\right)=\frac{\partial B\left(m^{S *}, \eta\right)}{\partial m^{S}} .
$$

Then, the principal receives

$$
\Pi^{D}\left(m^{S *}, \bar{\varphi}, \eta\right)=\frac{1}{4}\|\boldsymbol{\mu}\|^{2} \cos ^{2} \varphi\left(m^{S *}, \bar{\varphi}\right)-B\left(m^{S *}, \eta\right) .
$$

Proof See appendix.

Consider first the supervisor's expected bonus $B\left(m^{S *}, \eta\right)$ which comprises the optimal alignment to induce $m^{S *}$. Observe that the expected bonus is characterized by the likelihood ratio $\rho^{\prime}\left(m^{S}\right) / \rho\left(m^{S}\right)$ which, according to Holmström [1979], measures the principal's propensity to expect that the supervisor has not implemented the anticipated measurement intensity $m^{S *}$ when the favorable signal $M=1$ is realized. The likelihood ratio therefore measures the precision of the supervisor's performance evaluation. Finally observe that $B\left(m^{S *}, \eta\right)$ consists of the supervisor's relative measurement efficiency parameterized by $\eta$. The less efficient the supervisor's information acquisition is, characterized by a higher $\eta$, the higher must be the expected bonus payment $B(\cdot)$ in order to motivate the implementation of an arbitrary measurement intensity. 
Proposition 6 The supervisor extracts a rent for $m^{S *}>0$, which is increasing in $m^{S *}$.

Proof The supervisor obtains a rent if $R\left(m^{S *}, \eta\right) \equiv B\left(m^{S *}, \eta\right)-\eta C\left(m^{S *}\right)>0$. Suppose first that $m^{S}=0$. Since $\rho(0)=0$, this implies that $B(0, \eta)=0$, and consequently, $R(0, \eta)=0$. In order to demonstrate that $R\left(m^{S *}, \eta\right)>0$ for $m^{S *}>0$, we can use the first derivative of $R(\cdot)$ with respect to $m^{S}$, which leads after re-arranging to

$$
\frac{\partial R(\cdot)}{\partial m^{S}}=\frac{\rho\left(m^{S}\right) \eta C^{\prime \prime}\left(m^{S}\right)}{\rho^{\prime}\left(m^{S}\right)}+\frac{\rho\left(m^{S}\right) \eta C^{\prime}\left(m^{S}\right)\left(-\rho^{\prime \prime}\left(m^{S}\right)\right)}{\left[\rho^{\prime}\left(m^{S}\right)\right]^{2}} .
$$

Observe that $\partial R(\cdot) / \partial m^{S}>0$ for all $m^{S}>0$. Since $R(0, \eta)=0$ and $\partial R(\cdot) / \partial m^{S}>0$, it follows that $R(\cdot)>0$ for all $m^{S *}>0$.

Q.E.D.

Since her liability limit constraint (43) is binding, the supervisor extracts a rent. This is because the principal cannot impose negative transfers to expropriate the supervisor's rent. However, despite the supervisor's rent extraction, it cannot be inferred that delegation is always less beneficial from the principal's perspective than a centralized investment. In particular, this depends on the supervisor's cost parameter $\eta$, which characterizes her comparative advantage in acquiring the desired measures about the agent's performance. If this relative advantage is sufficiently high (low $\eta$ ), the expected bonus for inducing an arbitrary measurement intensity can be eventually less than the principal's required investment. In contrast, employing a supervisor with only a slight comparative advantage can impose higher costs for an arbitrary measurement intensity due to her rent extraction.

Next, consider the optimality condition for $m^{S *}$ emphasized by proposition 5 . The optimal measurement intensity $m^{S *}$ implicitly depends on two parameters: $(i)$ the congruity measure $\bar{\varphi}$ of the costless information system $\bar{S}$; and (ii), the supervisor's relative measurement efficiency $\eta$. Since both parameters determine the optimal measurement intensity $m^{S *}$, they implicitly affect the congruity of the agent's performance evaluation, and consequently, the efficiency of her effort allocation across tasks.

Proposition 7 The optimal measurement intensity $m^{S *}(\bar{\varphi}, \eta)$ is increasing in $\bar{\varphi}$ and decreasing in $\eta$. Overall, the principal's expected profit $\Pi^{D}\left(m^{S *}(\bar{\varphi}, \eta), \bar{\varphi}, \eta\right)$ is decreasing in $\bar{\varphi}$ and in $\eta$.

Proof See appendix. 
The first part of this result is obvious because a less congruent costless information system induces more distortion in the agent's effort allocation. In order to restrict this inefficiency, it is optimal to provide the supervisor with more powerful incentives aimed at motivating the implementation of a higher measurement intensity $m^{S *}$. Note that this directly leads to a higher rent extraction by the supervisor and by the agent. However, improving the congruity of the information system imposes convex increasing costs $B(\cdot)$. Thus, the additional inefficiency of an increasing $\bar{\varphi}$ cannot be perfectly compensated by inducing a higher intensity $m^{S *}(\cdot)$. As a consequence, $\Pi^{D}(\cdot)$ decreases in $\bar{\varphi}$. Moreover, it is more costly for the principal to motivate an arbitrary measurement intensity if the supervisor provides a less relative measurement efficiency (higher $\eta$ ). In this case, it is optimal to induce a lower measurement intensity, contemporaneously implying that the principal receives a lower expected profit.

\section{When is Delegation Profitable?}

According to previous observations, one crucial factor for the principal's preference in term of centralizing or delegating the information acquisition is the supervisor's relative measurement efficiency $\eta$. If $\eta$ is sufficiently low, one can expect that the principal favors delegation, and vice versa. However, it is not obvious how the congruity of the costless information system $\bar{\varphi}$ affects the profitability of delegation relative to a centralized investment. The purpose of this section is therefore to investigate, how the relationship between the congruity of the costless information system $\bar{\varphi}$ and the supervisor's relative measurement efficiency $\eta$ affect the optimal organizational design.

Before turning to the principal's preference for a particular organizational design aimed at efficiently improving the information system, it is first necessary to compare the optimal measurement intensities for both considered alternatives. Intuitively, one could expect that the supervisor establishes a more congruent information system if delegation is more profitable than centralization. Note, however, this is not in general true. It particularly depends on the shape of the supervisor's expected bonus $B(\cdot)$ characterized by the inverted likelihood ratio $\rho\left(m^{S}\right) / \rho^{\prime}\left(m^{S}\right)$. To identify whether or not $m^{S *}(\cdot)>m^{*}(\cdot)$, suppose the supervisor would implement the same measurement intensity as the principal does in the optimum, i.e. $m^{S}=m^{*}(\cdot)$. Then, the supervisor's optimal measurement intensity $m^{S *}(\cdot)$ is higher than the principal's optimal intensity $m^{*}(\cdot)$ if her expected bonus is less increasing in the point $m^{S}=m^{*}(\cdot)$ than the principal's investment costs. Formally, 
$m^{S *}(\cdot)>m^{*}(\cdot)$ if

$$
\frac{\partial B\left(m^{*}(\cdot), \eta\right)}{\partial m^{S}}<C^{\prime}\left(m^{*}(\cdot)\right)
$$

I demonstrate in the appendix that this is equivalent to

$$
\frac{\rho\left(m^{*}\right)}{\rho^{\prime}\left(m^{*}\right)}\left[\frac{C^{\prime \prime}\left(m^{*}\right)}{C^{\prime}\left(m^{*}\right)}-\frac{\rho^{\prime \prime}\left(m^{*}\right)}{\rho^{\prime}\left(m^{*}\right)}\right]<\frac{1-\eta}{\eta},
$$

where $m^{*} \equiv m^{*}(\cdot)$. If the inverted likelihood ratio $\rho(\cdot) / \rho^{\prime}(\cdot)$ is sufficiently low in $m^{*}$ and the supervisor provides an adequate relative measurement efficiency (low $\eta$ ), it is optimal from the principal's perspective to motivate a higher measurement intensity under delegation than it would be optimal for a centralized investment. In this case, delegation leads to the provision of more congruent incentives than centralization, thereby motivating the agent to implement less distorted effort. In general, the principal's decision on whether to centralize or to delegate the information acquisition depends, besides the precision of the supervisor's performance evaluation $\rho\left(m^{S}\right) / \rho^{\prime}\left(m^{S}\right)$, on the congruity of the costless information system $\bar{\varphi}$, and on the supervisor's relative measurement efficiency $\eta$.

Proposition 8 There exists a cut off cost parameter $\eta^{*} \in(0,1)$ implying that the principal is indifferent between centralization and delegation. Moreover, $\eta^{*}$ is strictly increasing (decreasing) in $\bar{\varphi}$ if $m^{S *}(\cdot)>(\leq) m^{*}(\cdot)$.

Proof See appendix.

Delegating the information acquisition to the supervisor is only favored by the principal if the supervisor provides a sufficient comparative advantage in measuring the agent's performance, which countervails her rent extraction. More interestingly, however, is the observation that the supervisor's minimum required measurement efficiency is lower (higher $\eta^{*}$ ), the less congruent the costless information system is (higher $\bar{\varphi}$ ). This applies when the supervisor's performance evaluation is sufficiently precise such that delegation leads to a higher measurement intensity than centralization. If in contrast the supervisor's performance evaluation is sufficiently imprecise such that $m^{S *}(\cdot) \leq m^{*}(\cdot)$, the reversed observation obtains.

The preceding observations are illustrated in figure 2, where the supervisor's relative measurement efficiency $\eta$ is depicted on the horizontal axis, and the principal expected profits for centralization and delegation are represented on the vertical axis. Since $\Pi^{C}(\cdot)$ is independent of $\eta$, its curve is parallel to the horizontal axis, whereas $\Pi^{D}(\cdot)$ is decreasing in $\eta$, see proposition 7 . The 


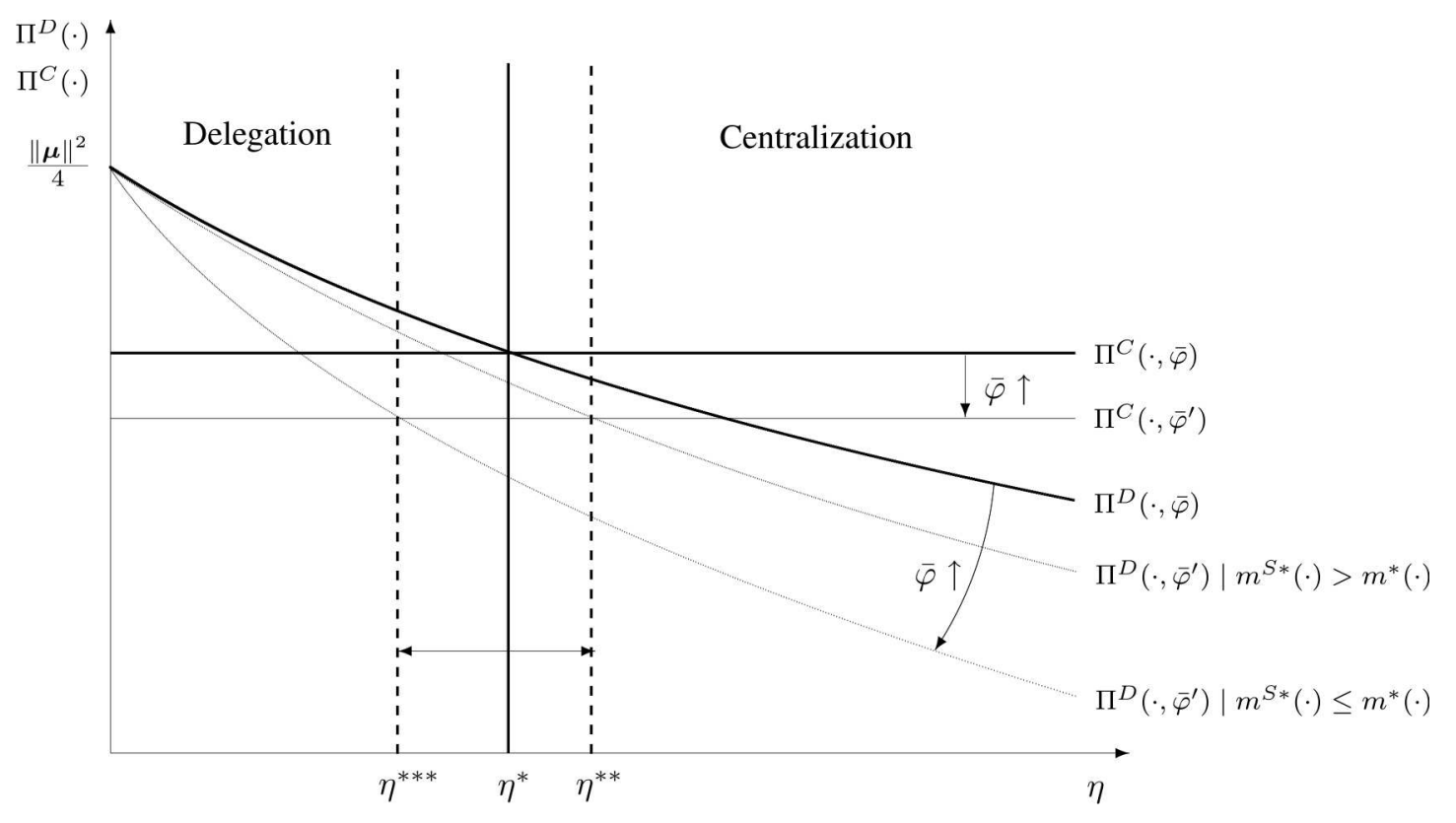

Figure 2: The Optimal Organizational Design

intersection of both curves characterizes the cut off $\eta^{*}$, where the principal is indifferent between centralization and delegation. For every $\eta<\eta^{*}$, the principal delegates the information acquisition to the supervisor, and centralizes, otherwise. Now suppose that the costless information system becomes more incongruent, i.e. $\bar{\varphi}$ increases to $\bar{\varphi}^{\prime}$. As a result, it is optimal to conduct a higher measurement intensity under centralization and delegation in order to mitigate the agent's effort distortion, see proposition 4 and 7. However, enhancing the measurement intensity imposes convex increasing costs, thereby implying that $\Pi^{C}(\cdot)$ and $\Pi^{D}(\cdot)$ decrease. Observe that the decline of $\Pi^{D}(\cdot)$ in $\bar{\varphi}$ particularly depends on the value of $\eta$, and on whether or not $m^{S *}(\cdot)>m^{*}(\cdot)$. Suppose for a moment that $m^{S *}(\cdot)>m^{*}(\cdot)$. If $\eta \leq \eta^{*}$, the marginal costs for enhancing the measurement intensity are lower for delegation than for centralization. As a consequence, $\Pi^{D}(\cdot)$ is less decreasing in $\bar{\varphi}$ than $\Pi^{C}(\cdot)$ for $\eta \leq \eta^{*}$. Graphically, this implies that $\eta^{*}$ increases to $\eta^{* *}$. Generally speaking, the supervisor's minimum required relative measurement efficiency for delegation to be superior, is lower, the less congruent the costless information system is. In contrast, a more congruent information system imposes higher requirements on the supervisor's relative measurement efficiency for delegation to be favored by the principal. However, if $m^{S *}(\cdot) \leq m^{*}(\cdot)$, the reverse is true such that $\eta^{*}$ decreases to $\eta^{* * *}$.

To illustrate the relevance of the preceding observations for the design of organizations, consider a firm with two departments $A$ and $B$, where the costless information system about employ- 
ees' effort allocation is assumed to be more congruent in department $A$ than in $B$. For example, department $A$ can be the sales department and $B$ the human resources department. For simplicity, assume that there is only one costless performance measure in each department available: $(i)$ the achieved sales in the sales department; and ( $i i)$, the office-hours in the human resources department. The achieved sales can thereby be inferred to be a more congruent performance measure than the office-hours in the human resources department. Suppose the supervisor's performance evaluation is sufficiently precise in both departments, thereby implying $m^{S *}(\cdot)>m^{*}(\cdot)$. The reversed argumentation applies for $m^{S *}(\cdot) \leq m^{*}(\cdot)$. According to previous results, it is optimal to conduct a higher measurement intensity in department $B$ than in department $A$ aimed at improving the respective information system and hence, mitigating effort distortion. Furthermore, the minimum required measurement efficiency a potential supervisor has to provide, is higher for department $A$ than for department $B$. This eventually leads to the conclusion that delegation for department $B$ (human resources department) is more likely than for department $A$ (sales department) since potential supervisors presumably meet the requirements with respect to their measurement efficiency for department $B$, rather than for department $A$.

The previous analysis reveals that job characteristics, particulary the congruity of costless performance measures, determine how costly performance measurement will be organized. Particularly, if the supervisor's performance evaluation is adequately precise, the previous analysis provides two crucial implications to explain why we can observe different practises for costly performance measurement even within the same organization. First, a sufficiently incongruent costless information system generally leads to delegation as the superior alternative. The rationale for this observation is that delegation - to be preferred by the principal-requires only a low relative measurement efficiency which is more likely to be provided by a potential supervisor. Second, the more congruent the costless information system is, the more likely is a centralized investment in the information acquisition. This can be observed because delegation-in order to be the more profitable alternative-would impose high requirements on the supervisor's relative measurement efficiency, which is less likely to be achieved by a potential supervisor. The reverse inference applies if the supervisor's performance evaluation is sufficiently imprecise. 


\section{Conclusion}

In economic relationships that are subject to moral hazard, objective performance measurement is frequently utilized to provide agents with incentives. However, applying performance measures in incentive contracts may motivate agents to implement inefficient effort allocations across relevant tasks. This occurs if an available performance evaluation does not perfectly reflect the agent's contribution to firm value. Then, it is desirable from the perspective of firms to use mechanisms which are suitable to mitigate this inefficiency. This paper investigates the costly acquisition of additional performance measures as one mechanism to alleviate effort distortion. The main emphasis is on the principal's preference for either centrally investing in assets that can be utilized to measure the agent's performance, or delegating the acquisition of additional measures to a supervisor.

This paper demonstrates that the less congruent the costless information system is, the greater is the investment in its improvement aimed at restricting effort distortion. This applies for both considered alternatives, i.e. for the centralized investment and for the delegation of the performance measurement to the supervisor. However, enhancing the congruity of the agent's performance evaluation does not only improve her effort allocation, but also provides her with a higher rent due to her liability limit.

The analysis indicates that employing a financially constrained supervisor for measuring the agent's performance can only be beneficial if she provides a sufficient relative measurement efficiency, which countervails her rent extraction. This paper illustrates that the profitability of delegation depends on three factors: $(i)$ the precision of the supervisor's evaluation system, $(i i)$ the supervisor's comparative cost advantage in obtaining the required information; and (iii), the congruence of the costless available information system about the agent's effort. Particularly, if the supervisor's performance evaluation is sufficiently precise, this investigation leads to the subsequent two implications. First, the principal generally prefers to delegate the information acquisition to the supervisor if the costless information system is sufficiently incongruent. This is because a less congruent costless information system imposes lower requirements on the supervisor's relative measurement efficiency, which is more likely to be satisfied by a potential supervisor. Second, the

principal favors in general a centralized investment if the costless information system is sufficiently congruent. This can be observed because delegation-in order to be the superior strategy-imposes a high requirement on the supervisor's relative measurement efficiency, which is less likely to be achievable by a potential supervisor. The reverse can be observed if the supervisor's performance 
evaluation is sufficiently imprecise.

This paper contributes to previous multi-task agency literature by shedding light on two potential alternatives for the costly generation of additional measures about the agent's effort aimed at improving the efficiency of her effort allocation. However, our understanding of how organizations respond to induced effort distortion is far from complete. There are several issues which are promising to consider in more detail. First, there exists empirical evidence that non-linear compensation schemes provoke individuals not only to distort their effort among different tasks, but also to distort their effort over time. ${ }^{20}$ Accordingly, besides motivating an efficient effort intensity and allocation across tasks, the additional objective of performance measurement is to motivate an efficient effort allocation over time. Furthermore, this paper focussed on the improvement of an information system in a setting with risk-neutral parties. The next logical step is to elaborate on the information acquisition in a setting with a risk-averse agent. In this case, the purpose of acquiring additional performance measures is not only restricted to improve her effort allocation, but also to reduce incentive risk. By incorporating a risk-averse agent in the underlying framework, prospective research may focus on this additional trade-off. However, I leave these important issues to future research.

${ }^{20}$ See e.g. Asch [1990] and Oyer [1998]. 


\section{Appendix}

\section{Proof of Lemma 1.}

As emphasized, effort distortion refers to the relation of $\boldsymbol{e}^{*}$ to $\boldsymbol{\mu}$. Hence, it can be characterized by the vector product $\boldsymbol{\mu}^{t} \boldsymbol{e}^{*}=\beta^{A} \boldsymbol{\mu}^{t} \overline{\boldsymbol{\omega}}$. Applying the relation for vector products gives $\boldsymbol{\mu}^{t} \boldsymbol{e}^{*}=$ $\beta^{A}\|\boldsymbol{\mu}\|\|\overline{\boldsymbol{\omega}}\| \cos \bar{\varphi}$, where $\|\cdot\|$ denotes the length of the respective vector, and $\bar{\varphi}$ the angle between vector $\boldsymbol{\mu}$ and vector $\overline{\boldsymbol{\omega}}$. As demonstrated in Thiele [2006], $\|\boldsymbol{\mu}\|,\|\overline{\boldsymbol{\omega}}\|$ and $\beta^{A}$ determine the effort intensity but do not influence the relative effort allocation. In contrast, $\bar{\varphi}$ measures effort distortion. To illustrate that $\bar{\varphi}$ measures also the efficiency loss imposed by the application of $\bar{S}$ in the agent's incentive contract, suppose first that $\overline{\boldsymbol{\omega}}=\xi^{-1} \boldsymbol{\mu}, \xi>0$, such that $\mathrm{E}[\bar{S} \mid \boldsymbol{e}]=\xi^{-1} \boldsymbol{\mu}^{t} \boldsymbol{e}$, i.e. the expected signal is congruent with the expected firm value. Then, the principal's expected profit on the basis of a congruent information system becomes $\hat{\Pi}=\boldsymbol{\mu}^{t} \boldsymbol{\mu} / 4$. Accordingly, $\Delta \Pi \equiv \hat{\Pi}-\Pi^{*}$ is the expected loss if $\bar{S}$ is not perfectly congruent. Consequently,

$$
\Delta \Pi=\frac{1}{4}\left[\boldsymbol{\mu}^{t} \boldsymbol{\mu}-\frac{\left(\boldsymbol{\mu}^{t} \overline{\boldsymbol{\omega}}\right)^{2}}{\overline{\boldsymbol{\omega}}^{t} \overline{\boldsymbol{\omega}}}\right]
$$

which is equivalent to

$$
\Delta \Pi=\frac{\left(\sum_{i} \mu_{i}^{2}\right)\left(\sum_{i} \bar{\omega}_{i}^{2}\right)-\left(\sum_{i} \mu_{i} \bar{\omega}_{i}\right)^{2}}{4 \sum_{i} \bar{\omega}_{i}^{2}} .
$$

According to the Cauchy-Schwarz inequality, $\Delta \Pi \geq 0$. Applying the relations $\sqrt{\sum_{i} \mu_{i}^{2}}=\|\boldsymbol{\mu}\|$ and $\sum_{i} \mu_{i} \bar{\omega}_{i}=\|\boldsymbol{\mu}\|\|\overline{\boldsymbol{\omega}}\| \cos \bar{\varphi}$ gives

$$
\Delta \Pi=\frac{1}{4}\|\boldsymbol{\mu}\|^{2}\left(1-\cos ^{2} \bar{\varphi}\right) \geq 0,
$$

which can be re-arranged to $\cos \bar{\varphi} \leq 1$. Consequently, $\bar{\varphi}$ measures $\Delta \Pi$. Finally, $\bar{\varphi} \in[0, \pi / 2]$ since $\mu_{i}, \bar{\omega}_{i} \geq 0, i=1, \ldots, n$.

Q.E.D.

\section{Proof of Proposition 4.}

To identify the effect of $\bar{\varphi}$ on $m^{*}$, define

$$
F \equiv \frac{1}{4}\|\boldsymbol{\mu}\|^{2} \sin \left(2 \varphi\left(m^{*}, \bar{\varphi}\right)\right)\left(-\varphi_{m}\right)-C^{\prime}\left(m^{*}\right)=0 .
$$

Applying the Implicit Function Theorem gives $d m^{*} / d \bar{\varphi}=-(\partial F / \partial \bar{\varphi}) /(\partial F / \partial m)$. Since the second-order condition for $m^{*}$ is satisfied, it follows $\partial F / \partial m<0$. Moreover,

$$
\frac{\partial F}{\partial \bar{\varphi}}=\frac{1}{4}\|\boldsymbol{\mu}\|^{2}\left[2 \cos (2 \varphi(\cdot))\left(-\varphi_{m}\right) \varphi_{\bar{\varphi}}+\sin (2 \varphi(\cdot))\left(-\varphi_{m \bar{\varphi}}\right)\right],
$$


which is strictly positive for all $\varphi(\cdot) \in(0, \pi / 4)$. Consequently, $d m^{*} / d \bar{\varphi}>0$. To identify the effect of $\bar{\varphi}$ on $\Pi^{C}(\cdot)$, one can apply the Envelope Theorem, which gives

$$
\frac{d \Pi^{C}\left(m^{*}(\bar{\varphi}), \bar{\varphi}\right)}{d \bar{\varphi}}=-\frac{1}{4}\|\boldsymbol{\mu}\|^{2} \sin (2 \varphi(\cdot)) \varphi_{\bar{\varphi}}
$$

Observe that $d \Pi^{C}(\cdot) / d \bar{\varphi}<0$ for all $\varphi(\cdot) \in(0, \pi / 4)$.

Q.E.D.

\section{Proof of Proposition 5.}

First observe that (41) is equivalent to

$$
\beta^{S}\left(m^{S}, \eta\right)=\frac{\eta C^{\prime}\left(m^{S}\right)}{\rho^{\prime}\left(m^{S}\right)}
$$

with $\beta^{S}(\cdot)$ as the required bonus to induce an arbitrary $m^{S}$. Consequently, the expected bonus $B\left(m^{S}, \eta\right)=\beta^{S}\left(m^{S}, \eta\right) \rho\left(m^{S}\right)$ becomes

$$
B\left(m^{S}, \eta\right)=\frac{\eta C^{\prime}\left(m^{S}\right) \rho\left(m^{S}\right)}{\rho^{\prime}\left(m^{S}\right)} .
$$

Note that $\beta^{S}(\cdot)$ needs to be strictly positive in order to induce $m^{S}>0$. Consequently, (42) is satisfied as long as (43) holds and therefore omits. The Lagrangian therefore becomes

$$
\begin{aligned}
\mathcal{L}\left(\alpha^{S}, m^{S}\right)= & \frac{1}{4}\|\boldsymbol{\mu}\|^{2} \cos ^{2} \varphi\left(m^{S}, \bar{\varphi}\right)-\alpha^{S}-B\left(m^{S}, \eta\right) \\
& +\lambda\left[\alpha^{S}+B\left(m^{S}, \eta\right)-\eta C\left(m^{S}\right)\right]+\xi \alpha^{S} .
\end{aligned}
$$

The first-order conditions with respect to $\alpha^{S}$ and $m^{S}$ are

$$
\begin{gathered}
-1+\lambda+\xi=0 \\
\frac{1}{4}\|\boldsymbol{\mu}\|^{2} \sin \left(2 \varphi\left(m^{S}, \bar{\varphi}\right)\right)\left(-\varphi_{m}\right)-\frac{\partial B(\cdot)}{\partial m^{S}}+\lambda\left[\frac{\partial B(\cdot)}{\partial m^{S}}-\eta C^{\prime}\left(m^{S}\right)\right]=0
\end{gathered}
$$

and the complementary slackness conditions,

$$
\begin{gathered}
\lambda\left[\alpha^{S}+B(\cdot)-\eta C\left(m^{S}\right)\right]=0, \\
\xi \alpha=0 .
\end{gathered}
$$

To find a solution of this problem, suppose for a moment that $\lambda>0$. Note that in this case, $\alpha^{S}+B(\cdot)-\eta C\left(m^{S}\right)=0$ due to (61). Since it is required that $\alpha^{S} \geq 0$, this would imply that $B(\cdot) \leq$ 
$\eta C\left(m^{S}\right)$. In this case, the supervisor maximizes her expected utility by choosing $m^{S}=0$. Hence, $\lambda>0$ cannot be a solution of this problem. Consequently, $\lambda=0$, i.e. the supervisor's participation constraint is not binding. We can further infer from (59) that $\xi=1$. Thus, in accordance to (62), the principal sets $\alpha^{S *}=0$. Since $\lambda=0$, we can observe from (60) that $m^{S *}$ solves

$$
\frac{1}{4}\|\boldsymbol{\mu}\|^{2} \sin \left(2 \varphi\left(m^{S *}, \bar{\varphi}\right)\right)\left(-\varphi_{m}\right)=\frac{\partial B\left(m^{S *}, \eta\right)}{\partial m^{S}} .
$$

Next, it is necessary to proof that the first-order approach is also sufficient. Recall from assumption 2 that the gross payoff is concave increasing in $m^{S}$. Consequently, it is sufficient to show that $B(\cdot)$ is convex increasing in $m^{S}$. The first derivative of $B(\cdot)$ with respect to $m^{S}$ gives

$$
\frac{\partial B(\cdot)}{\partial m^{S}}=\eta C^{\prime}(\cdot)+\frac{\rho\left(m^{S}\right) \eta C^{\prime \prime}\left(m^{S}\right)}{\rho^{\prime}\left(m^{S}\right)}+\frac{\rho\left(m^{S}\right) \eta C^{\prime}\left(m^{S}\right)\left(-\rho^{\prime \prime}\left(m^{S}\right)\right)}{\left[\rho^{\prime}\left(m^{S}\right)\right]^{2}},
$$

which is strictly positive for all $m^{S}>0$, i.e. $B(\cdot)$ is increasing in $m^{S}$. The second derivative leads to

$$
\begin{aligned}
\frac{\partial^{2} B(\cdot)}{\partial\left(m^{S}\right)^{2}}= & \eta C^{\prime \prime}(\cdot)+\frac{\left[\rho^{\prime}(\cdot) \eta C^{\prime \prime}(\cdot)+\rho(\cdot) \eta C^{\prime \prime \prime}(\cdot)\right] \rho^{\prime}(\cdot)-\rho(\cdot) \eta C^{\prime \prime}(\cdot) \rho^{\prime \prime}(\cdot)}{\left[\rho^{\prime}(\cdot)\right]^{2}} \\
& +\frac{\left[\left(\rho^{\prime}(\cdot) \eta C^{\prime}(\cdot)+\rho(\cdot) \eta C^{\prime \prime}(\cdot)\right)\left(-\rho^{\prime \prime}(\cdot)\right)\right]\left[\rho^{\prime}(\cdot)\right]^{2}}{\left[\rho^{\prime}(\cdot)\right]^{4}} \\
& -\frac{2 \rho(\cdot) \eta C^{\prime}(\cdot)\left(-\rho^{\prime \prime}(\cdot)\right) \rho^{\prime}(\cdot) \rho^{\prime \prime}(\cdot)}{\left[\rho^{\prime}(\cdot)\right]^{4}}
\end{aligned}
$$

which can be re-arranged to

$$
\begin{aligned}
\frac{\partial^{2} B(\cdot)}{\partial\left(m^{S}\right)^{2}}= & 2 \eta C^{\prime \prime}(\cdot)+\frac{\rho(\cdot) \eta C^{\prime \prime \prime}(\cdot)}{\rho^{\prime}(\cdot)}+\frac{\rho(\cdot) \eta C^{\prime \prime}(\cdot)\left(-\rho^{\prime \prime}(\cdot)\right)}{\left[\rho^{\prime}(\cdot)\right]^{2}} \\
& +\frac{\left[\rho^{\prime}(\cdot) \eta C^{\prime}(\cdot)+\rho(\cdot) \eta C^{\prime \prime}(\cdot)\right]\left[-\rho^{\prime \prime}(\cdot)\right]}{\left[\rho^{\prime}(\cdot)\right]^{2}}+\frac{2 \rho(\cdot) \eta C^{\prime}(\cdot)\left(-\rho^{\prime \prime}(\cdot)\right)^{2}}{\left[\rho^{\prime}(\cdot)\right]^{3}}
\end{aligned}
$$

Observe that $\partial^{2} B(\cdot) / \partial\left(m^{S}\right)^{2}>0$. Accordingly, $B(\cdot)$ is convex increasing in $m^{S}$. This implies that the first-order approach to identify the optimal measurement intensity is also sufficient. Finally, substituting $\alpha^{S *}=0$ and $m^{S *}$ in the principal's objective function gives $\Pi^{D}\left(m^{S *}, \bar{\varphi}, \eta\right)$.

Q.E.D.

\section{Proof of Proposition 7.}

For identifying $d m^{S *} / d \bar{\varphi}$, define

$$
F \equiv \frac{1}{4}\|\boldsymbol{\mu}\|^{2} \sin \left(2 \varphi\left(m^{*}, \bar{\varphi}\right)\right)\left(-\varphi_{m}\right)-\frac{\partial B(\cdot)}{\partial m^{S}}=0 .
$$


The Implicit Function Theorem gives $d m^{S *} / d \bar{\varphi}=-(\partial F / \partial \bar{\varphi}) /\left(\partial F / \partial m^{S}\right)$. First, one get

$$
\frac{\partial F}{\partial \bar{\varphi}}=\frac{1}{4}\|\boldsymbol{\mu}\|^{2}\left[2 \cos (2 \varphi(\cdot))\left(-\varphi_{m}\right) \varphi_{\bar{\varphi}}+\sin (2 \varphi(\cdot))\left(-\varphi_{m \bar{\varphi}}\right)\right]
$$

which is strictly positive for all $m^{S}>0$ and $\bar{\varphi} \in[0, \pi / 2)$. Since the second-order condition for $m^{S *}$ is satisfied, we have $\partial F / \partial m^{S}<0$. Consequently, $d m^{S *} / d \bar{\varphi}>0$.

The effect of $\eta$ on $m^{S *}$ can be shown by applying $d m^{S *} / d \eta=-(\partial F / \partial \eta) /\left(\partial F / \partial m^{S}\right)$. Recall that $\partial F / \partial m^{S}<0$. Furthermore, the first derivative of $F$ with respect to $\eta$ yields

$$
\frac{\partial F}{\partial \eta}=-C^{\prime}\left(m^{S}\right)-\frac{\rho\left(m^{S}\right) C^{\prime \prime}\left(m^{S}\right)}{\rho^{\prime}\left(m^{S}\right)}-\frac{\rho\left(m^{S}\right) C^{\prime}\left(m^{S}\right)\left(-\rho^{\prime \prime}\left(m^{S}\right)\right)}{\left[\rho^{\prime}\left(m^{S}\right)\right]^{2}},
$$

which is strictly negative for all $m^{S}>0$. Consequently, $d m^{S *} / d \eta<0$. To illustrate the effect of $\eta$ on $\Pi^{D}(\cdot)$, one can apply the Envelope Theorem, which gives

$$
\frac{d \Pi^{D}(\cdot)}{d \eta}=-\frac{\partial B(\cdot)}{\partial \eta}=-\frac{C^{\prime}\left(m^{S}\right) \rho\left(m^{S}\right)}{\rho^{\prime}\left(m^{S}\right)} .
$$

Thus, $d \Pi^{D}(\cdot) / d \eta<0$ for all $m^{S *}>0$.

Q.E.D.

\section{Comparison of Measurement Intensities.}

First, recall that

$$
\frac{\partial B(\cdot)}{\partial m^{S}}=\eta C^{\prime}(\cdot)+\frac{\rho\left(m^{S}\right) \eta C^{\prime \prime}\left(m^{S}\right)}{\rho^{\prime}\left(m^{S}\right)}+\frac{\rho\left(m^{S}\right) \eta C^{\prime}\left(m^{S}\right)\left(-\rho^{\prime \prime}\left(m^{S}\right)\right)}{\left[\rho^{\prime}\left(m^{S}\right)\right]^{2}} .
$$

Consequently, $C^{\prime}\left(m^{*}(\cdot)\right)>\partial B\left(m^{*}(\cdot), \eta\right) / \partial m^{S}$ is equivalent to

$$
\frac{1-\eta}{\eta} C^{\prime}\left(m^{*}(\cdot)\right)>\frac{\rho\left(m^{*}(\cdot)\right) C^{\prime \prime}\left(m^{*}(\cdot)\right)}{\rho^{\prime}\left(m^{*}(\cdot)\right)}+\frac{\rho\left(m^{*}(\cdot)\right) C^{\prime}\left(m^{*}(\cdot)\right)\left[-\rho^{\prime \prime}\left(m^{*}(\cdot)\right)\right]}{\left[\rho^{\prime}\left(m^{*}(\cdot)\right)\right]^{2}} .
$$

This can be transformed to

$$
\frac{1-\eta}{\eta}>\frac{C^{\prime \prime}\left(m^{*}(\cdot)\right)}{C^{\prime}\left(m^{*}(\cdot)\right)} \frac{\rho\left(m^{*}(\cdot)\right)}{\rho^{\prime}\left(m^{*}(\cdot)\right)}+\frac{\rho\left(m^{*}(\cdot)\right)\left[-\rho^{\prime \prime}\left(m^{*}(\cdot)\right)\right]}{\left[\rho^{\prime}\left(m^{*}(\cdot)\right)\right]^{2}},
$$

and hence,

$$
\frac{1-\eta}{\eta}>\frac{\rho\left(m^{*}(\cdot)\right)}{\rho^{\prime}\left(m^{*}(\cdot)\right)}\left[\frac{C^{\prime \prime}\left(m^{*}(\cdot)\right)}{C^{\prime}\left(m^{*}(\cdot)\right)}-\frac{\rho^{\prime \prime}\left(m^{*}(\cdot)\right)}{\rho^{\prime}\left(m^{*}(\cdot)\right)}\right]
$$

Q.E.D. 


\section{Proof of Proposition 8.}

Observe that $\eta^{*}$ implies $F \equiv \Pi^{D}\left(m^{S *}\left(\bar{\varphi}, \eta^{*}\right), \bar{\varphi}, \eta\right)-\Pi^{C}\left(m^{*}(\bar{\varphi}), \bar{\varphi}\right)=0$. Suppose first that $\eta \geq 1$. Due to rent extraction, inducing an arbitrary measurement intensity is more costly under delegation than under centralization. Accordingly, we have $B(m)>\eta C(m) \geq C(m)$ for all $m>0$ and $\eta \geq 1$. In contrast, if $\eta=0$, the supervisor can be induced to implement $m^{S} \rightarrow \infty$ with $B\left(m^{S}, 0\right)=0$. In this case, delegation is strictly superior. Moreover, recall from proposition 7 that $d \Pi^{D}(\cdot) / d \eta<0$ for all $m^{S *}>0$. Consequently, there exists a cut off $\eta^{*} \in(0,1)$ implying that the principal is indifferent between centralization and delegation.

To identify the effect of $\bar{\varphi}$ on $\eta^{*}$, one can apply the Implicit Function Theorem, which gives $d \eta^{*} / d \bar{\varphi}=-(\partial F / \partial \bar{\varphi}) /(\partial F / \partial \eta)$. In order to derive $\partial F / \partial \bar{\varphi}$, it useful to apply the Envelope Theorem for $\Pi^{C}(\cdot)$ and $\Pi^{D}(\cdot)$ separately. Thus,

$$
\frac{\partial \Pi^{D}(\cdot)}{\partial \bar{\varphi}}=-\frac{1}{4}\|\boldsymbol{\mu}\|^{2} \sin \left(2 \varphi\left(m^{S *}(\cdot), \bar{\varphi}\right)\right) \varphi_{\bar{\varphi}}\left(m^{S *}\right),
$$

and

$$
\frac{\partial \Pi^{C}(\cdot)}{\partial \bar{\varphi}}=-\frac{1}{4}\|\boldsymbol{\mu}\|^{2} \sin \left(2 \varphi\left(m^{*}(\cdot), \bar{\varphi}\right)\right) \varphi_{\bar{\varphi}}\left(m^{*}\right)
$$

Since

$$
\frac{\partial F}{\partial \bar{\varphi}}=\frac{\partial \Pi^{D}(\cdot)}{\partial \bar{\varphi}}-\frac{\partial \Pi^{C}(\cdot)}{\partial \bar{\varphi}}
$$

we have

$$
\begin{aligned}
\frac{\partial F}{\partial \bar{\varphi}}=\frac{\|\boldsymbol{\mu}\|^{2}}{4}[ & \left.\sin \left(2 \varphi\left(m^{*}(\cdot), \cdot\right)\right) \varphi_{\bar{\varphi}}\right|_{m^{*}(\cdot)} \\
& \left.-\left.\sin \left(2 \varphi\left(m^{S *}(\cdot), \cdot\right)\right) \varphi_{\bar{\varphi}}\right|_{m^{S *}(\cdot)}\right] .
\end{aligned}
$$

Observe that $\partial F / \partial \bar{\varphi}>0$ if

$$
\frac{\sin \left(2 \varphi\left(m^{*}(\cdot), \bar{\varphi}\right)\right)}{\sin \left(2 \varphi\left(m^{S *}(\cdot), \bar{\varphi}\right)\right)}>\frac{\varphi_{\bar{\varphi}}\left(m^{S *}(\cdot)\right)}{\varphi_{\bar{\varphi}}\left(m^{*}(\cdot)\right)} .
$$

Recall first that $\varphi\left(m^{S *}(\cdot), \bar{\varphi}\right)<\varphi\left(m^{*}(\cdot), \bar{\varphi}\right)$ if $m^{S *}(\cdot)>m^{*}(\cdot)$. Consequently,

$$
\sin \left(2 \varphi\left(m^{S *}(\cdot), \bar{\varphi}\right)\right)<\sin \left(2 \varphi\left(m^{*}(\cdot), \bar{\varphi}\right)\right)
$$

for $\varphi(\cdot) \in[0, \pi / 2]$. Moreover, $\left.\varphi_{\bar{\varphi}}\right|_{m^{*}(\cdot)}>\left.\varphi_{\bar{\varphi}}\right|_{m^{S *}(\cdot)}$. This eventually implies that (79) is satisfied for $m^{S *}(\cdot)>m^{*}(\cdot)$. Therefore, $\partial F / \partial \bar{\varphi}>0$ if $m^{S *}(\cdot)>m^{*}(\cdot)$. In contrast, $\partial F / \partial \bar{\varphi} \leq 0$ if 
$m^{S *}(\cdot) \leq m^{*}(\cdot)$. Finally observe that $\Pi^{C}(\cdot)$ is independent of $\eta$. Thus, the Envelope Theorem gives

$$
\frac{\partial F}{\partial \eta}=\frac{\partial \Pi^{D}(\cdot)}{\partial \eta}=-\frac{\partial B(\cdot)}{\partial \eta}=-\frac{\rho(\cdot) C^{\prime}(\cdot)}{\rho^{\prime}(\cdot)},
$$

which is strictly negative for all $m^{S *}(\cdot)>0$. Thus, $d \eta^{*} / d \bar{\varphi}>0$ if $m^{S *}(\cdot)>m^{*}(\cdot)$; and $d \eta^{*} / d \bar{\varphi} \leq$ 0 , otherwise.

Q.E.D. 


\section{References}

Asch, B. J. (1990). Do incentives matter? the case of navy recruiters. Industrial \& Labor Relations Review 43(3), 89-106.

Baker, G. (2000). The use of performance measures in incentive contracting. American Economic Review 90(2), 415-420.

Baker, G. (2002). Distortion and risk in optimal incentive contracts. Journal of Human Resources $37(4), 728-751$.

Baker, G., R. Gibbons, and K. J. Murphy (1994). Subjective performance measures in optimal incentive contracts. Quarterly Journal of Economics 109(4), 1125-1156.

Baker, G., R. Gibbons, and K. J. Murphy (2002). Relational contracts and the theory of the firm. Quarterly Journal of Economics 117(1), 39-84.

Banker, R. D. and A. Thevaranjan (2000). Goal congruence and evaluation of performance measures. Working Paper, University of Texas at Dallas.

Brickley, J. A. and J. L. Zimmerman (2001). Changing incentives in a multitask environment: Evidence from a top-tier business school. Journal of Corporate Finance 7, 367-396.

Christensen, P. O. and G. A. Feltham (2005). Economics of Accounting: Volume II - Performance Evaluation. New York: Springer.

Corts, K. S. (2005). Teams vs. individual accountability: Solving multi-task problems through job design. Working Paper, Univerity of Toronto.

Datar, S., S. C. Kulp, and R. A. Lambert (2001). Balancing performance measures. Journal of Accounting Research 39(1), 75-92.

Demougin, D. and C. Fluet (2001). Monitoring versus incentives. European Economic Review 45, $1741-1764$.

Feltham, G. A. and J. Xie (1994). Performance measure congruity and diversity in multi-task principal/agent relations. Accounting Review 69(3), 429-453.

Gibbons, R. (1998). Incentives in organizations. Journal of Economic Perspectives 12(4), 115-132. 
Gibbons, R. (2005). Incentives between firms (and within). Management Science 51(1), 2-17.

Gibbs, M., K. A. Merchant, W. A. Van der Stede, and M. E. Vargus (2004). Performance measure properties and incentives. IZA Discussion Paper No. 1356.

Holmström, B. (1979). Moral hazard and observability. Bell Journal of Economics 10(1), 74-91.

Holmström, B. (1999). The firm as a subeconomy. Journal of Law, Economics, \& Organization 15(1), 74-102.

Holmström, B. and P. Milgrom (1991). Multitask principal-agent analyses: Incentives contracts, asset ownership, and job design. Journal of Law, Economics and Organization 7, 24-52.

Hughes, J. S., L. Zhang, and J. Xie (2005). Production externalities, congruity of aggregate signals, and optimal task assignments. Contemporary Accounting Research 22(2), 393-408.

Kerr, S. (1975). On the folly of rewarding a, while hoping for b. Academy of Management Journal 18(4), 769-783.

Kim, S. K. (1997). Limited liability and bonus contracts. Journal of Economics \& Management Strategy 6(4), 899-913.

Kim, S. K. and Y. S. Suh (1991). Ranking of accounting information systems for management control. Journal of Accounting Research 29(2), 386-396.

Lambert, R. A. (2001). Contracting theory and accounting. Journal of Accounting and Economics 32(1), 3-87.

Milgrom, P. R. (1981). Good news and bad news: Representation theorems and applications. Bell Journal of Economics 12(2), 380-391.

Oyer, P. (1998). Fiscal year ends and nonlinear incentive contracts: The effect on business seasonality. Quarterly Journal of Economics 113(1), 149-185.

Park, E. S. (1995). Incentive contracting under limited liability. Journal of Economics \& Management Strategy 4(3), 477-490.

Pitchford, R. (1998). Moral hazard and limited liability: The real effects of contract bargaining. Economics Letters 61, 251-259. 
Prendergast, C. (1999). The provision of incentives in firms. Journal of Economic Literature 37(1), 7-63.

Prendergast, C. and R. H. Topel (1996). Favoritism in organizations. Journal of Political Economy 104(5), 958-978.

Schöttner, A. (2005). Relational contracts and job design. Working Paper Humboldt-University Berlin.

Thiele, V. (2006). Task-specific abilities in multi-task agency relations. Working Paper, University of British Columbia. 